December 8, 2008

\title{
Explicit solution of the (quantum) elliptic Calogero-Sutherland model
}

\author{
Edwin Langmann1 \\ Theoretical Physics, AlbaNova, SE-106 91 Stockholm, Sweden \\ Dedicated to the memory of Vadim Kuznetsov
}

\begin{abstract}
We derive explicit formulas for the eigenfunctions and eigenvalues of the elliptic Calogero-Sutherland model as infinite series, to all orders and for arbitrary particle numbers and coupling parameters. The eigenfunctions obtained provide an elliptic deformation of the Jack polynomials. We prove in certain special cases that these series have a finite radius of convergence in the nome $q$ of the elliptic functions, including the two particle (= Lamé) case for non-integer coupling parameters.
\end{abstract}

PACS: 02.30.Ik, 03.65.-w, 05.30.Pr

MSC-class: 81Q05, 35Q58

\section{Introduction}

In this paper we present an explicit solution of the elliptic generalization of a quantum many body model in one dimension which is usually associated with the names of Calogero and Sutherland [C1, Su1, C2] and whose quantum integrability was proved by Olshanetsky and Perelomov $\mathrm{OP}$. Our solution is based on a remarkable functional identity stated in Lemma 2.1.1 below. This identity was found in [L1, L2] using quantum field theory techniques [CL], but to make the present paper self-contained we also include an alternative, elementary proof of this key result. Our main results are explicit formulas for the eigenvalues and eigenfunctions of this model as infinite series. We also prove absolute convergence of these series in certain special cases.

\section{$1.1 \quad$ Background}

The elliptic Calogero-Sutherland (eCS) model is defined by the differential operator

$$
H \stackrel{\text { def }}{=}-\sum_{j=1}^{N} \frac{\partial^{2}}{\partial x_{j}^{2}}+\gamma \sum_{1 \leq j<k \leq N} V\left(x_{j}-x_{k}\right)
$$

\footnotetext{
${ }^{1}$ langmann@kth.se
} 
with $-\pi \leq x_{j} \leq \pi$ coordinates on the circle, $N=2,3, \ldots, \gamma>-1 / 2$, and the function

$$
V(r) \stackrel{\text { def }}{=} \sum_{m \in \mathbb{Z}} \frac{1}{4 \sin ^{2}[(r+\mathrm{i} \beta m) / 2]}=\wp(r \mid \pi, \mathrm{i} \beta / 2)+c_{0} \quad(\beta>0)
$$

with the Weierstrass elliptic function $\wp$ and the constant

$$
c_{0}=\frac{1}{12}-\sum_{m=1}^{\infty} \frac{1}{2 \sinh ^{2}[(\beta m) / 2]}=\frac{1}{12}-\sum_{n=1}^{\infty} \frac{2 q^{2 n}}{\left(1-q^{2 n}\right)^{2}}
$$

where

$$
q \stackrel{\text { def }}{=} \mathrm{e}^{-\beta / 2}
$$

(see Appendix A.1 for more details); we find it convenient to shift $\wp$ by a constant to simplify some formulas later on. We also introduce the function

$$
\theta(r) \stackrel{\text { def }}{=} \sin (r / 2) \prod_{n=1}^{\infty}\left(1-2 q^{2 n} \cos (r)+q^{4 n}\right)
$$

which is equal, up to a multiplicative constant, to the Jacobi Theta function $\vartheta_{1}(r / 2)$. This allows us to write

$$
V(r)=-\frac{d^{2}}{d r^{2}} \log \theta(r)
$$

(see Appendix A.1 for details). This differential operator $H$ defines a quantum mechanical model of $N$ identical particles moving on a circle of length $2 \pi$ and interacting with a two body potential $\gamma V(r)$ with $\gamma$ the coupling constant. To be more precise: the model we are interested in corresponds to a particularly 'nice' self-adjoint extension of this differential operator [KT] (which is essentially the Friedrichs extension [RS]), and we only consider eigenfunctions describing non-distinguishable particles. It is convenient to parametrize the coupling constant as follows,

$$
\gamma \stackrel{\text { def }}{=} 2 \lambda(\lambda-1)
$$

where $\lambda>1 / 2$ is the coupling parameter. Our restrictions on the parameters $q$ and $\lambda$ are for simplicity and since they include most of the cases where $H$ defines a self-adjoint operator bounded from below, but many of our results can be analytically continued to other complex parameter values.

In the limiting case $q=0(\beta \rightarrow \infty)$ we have $V(r)=(1 / 4) \sin ^{-2}(r / 2)$, and the differential operator $H$ in (1.1) reduces to the one defining a celebrated model solved a long time ago by Sutherland [Su1] who found explicit formulas for all eigenvalues and an algorithm to construct the corresponding eigenfunctions of $H$. The exact eigenfunction $\Psi_{\mathbf{n}}$ of the Sutherland model can be labeled by partitions $\mathbf{n}$, i.e. integer vectors $\mathbf{n}=\left(n_{1}, n_{2}, \ldots, n_{N}\right) \in$ $\mathbb{N}_{0}^{N}$ such that $n_{1} \geq n_{2} \geq \ldots \geq n_{N} \geq 0$, and they are of the form

$$
\Psi_{\mathbf{n}}(\mathbf{x})=\mathcal{J}_{\mathbf{n}}(\mathbf{z}) \Psi_{0}(\mathbf{x}), \quad z_{j} \stackrel{\text { def }}{=} \mathrm{e}^{\mathrm{i} x_{j}}
$$

with

$$
\Psi_{0}(\mathbf{x}) \stackrel{\text { def }}{=} \prod_{1 \leq j<k \leq N} \theta\left(x_{k}-x_{j}\right)^{\lambda}
$$


and the $\mathcal{J}_{\mathbf{n}}(\mathbf{z})$ symmetric polynomials with simple corresponding eigenvalues given in (2.13) below. The $\mathcal{J}_{\mathbf{n}}$ are known as Jack polynomials and are also of interest in combinatorics; see $[\mathrm{McD}, \mathrm{St}$.

It is interesting to note that, to get all eigenfunctions of interest in physics, one should multiply the r.h.s. of (1.8) by a factor $\mathrm{e}^{-\mathrm{i} P\left(x_{1}+\cdots+x_{N}\right)}$ with an arbitrary $P \in \mathbb{N}$ corresponding to an additional possible center-of-mass motion. One can account for this by extending the definition of the Jack polynomials to all integer vectors $\mathbf{n}$ such that $n_{1} \geq n_{2} \geq \cdots \geq n_{N}$ (with $n_{N}$ possibly negative) as follows,

$$
\mathcal{J}_{\boldsymbol{\lambda}-P \mathbf{e}}(\mathbf{z})=c_{\boldsymbol{\lambda}, P}\left(z_{1} z_{2} \cdots z_{N}\right)^{-P} \mathcal{J}_{\boldsymbol{\lambda}}(\mathbf{z}), \quad \mathbf{e} \stackrel{\text { def }}{=}(1,1, \cdots, 1)
$$

for all partitions $\boldsymbol{\lambda}$ and $P \in \mathbb{N}$; the $c_{\boldsymbol{\lambda}, P}$ are some non-zero constants depending on the normalization of the $\mathcal{J}_{\mathbf{n}}$. This equation actually holds true also for negative integers $P$; see e.g. [St]. It is common to ignore this point and restrict the $\mathbf{n}$ to partitions.

Sutherland's solution method is based on the fact that, for $q=0$, the differential operator $H$ in (1.1) has an exact eigenstate $\Psi_{0}(\mathbf{x})$ of a form as in (1.9). This is no longer true for $q>0$ [Su2], and thus Sutherland's approach cannot be generalized to the elliptic case. In this paper we elaborate another algorithm which allows to solve also the general elliptic case [L1, L5]. In the trigonometric limit $q=0$, this algorithm simplifies to one which is different from Sutherland's; see [L3] for a comparison of these algorithms. The crucial difference is that, while Sutherland computes the Jack polynomials $\mathcal{J}_{\mathbf{n}}(\mathbf{z})$ as linear combinations of the following basis in the space of symmetric polynomials, $M_{\boldsymbol{\lambda}}(\mathbf{z})=\sum_{\pi} \prod_{j=1}^{N} z_{j}^{\lambda_{\pi(j)}}$ for partitions $\boldsymbol{\lambda}$ and the sum over all distinct permutations $\pi$ in the permutation group $S_{N}$, we use a different generating set of functions $f_{\mathbf{n}}(\mathbf{z})$; see (2.9) for $\Theta(z)=(1-z)$. It is important to note that the $f_{\mathbf{n}}$ are well-defined and non-zero also for integer vectors $\mathbf{n}$ which are not partitions. Thus the $f_{\mathbf{n}}$ provide an overcomplete generating set in the space of symmetric polynomials. Expanding the $\mathcal{J}_{\mathbf{n}}$ in this generating set

$$
\mathcal{J}_{\mathbf{n}}(\mathbf{z})=\sum_{\mathbf{m}} \alpha_{\mathbf{n}}(\mathbf{m}) f_{\mathbf{m}}(\mathbf{z})
$$

the expansion coefficients $\alpha_{\mathbf{n}}(\mathbf{m})$ obey simple recursion relations [L3] which can be solved explicitly [L5].

We emphasize that, in our approach, we get eigenfunctions $\Psi_{\mathbf{n}}$ for all integer vectors $\mathbf{n}$. Obviously, if $\mathcal{E}_{\mathbf{n}}$ for a non-partition $\mathbf{n} \in \mathbb{Z}^{N}$ is different from all $\mathcal{E}_{\boldsymbol{\lambda}-P \mathbf{e}}$ for partitions $\boldsymbol{\lambda}$ and $P \in \mathbb{N}$, then the corresponding eigenfunction $\Psi_{\mathbf{n}}$ must vanish, otherwise it must be a linear combination of the $\Psi_{\boldsymbol{\lambda}-P \mathbf{e}}$ such that $\mathcal{E}_{\mathbf{n}}=\mathcal{E}_{\boldsymbol{\lambda}-P \mathbf{e}} 2$ It would be interesting to investigate this overcompleteness.

\subsection{The nature of our solution}

For $q>0$, our algorithm leads to eigenfunctions $\Psi_{\mathbf{n}}$ as in (1.8)-(1.10) with a natural generalization of the functions $f_{\mathbf{n}}(\mathbf{z})$ given in Proposition 2.2.1 below. It is natural to regard

\footnotetext{
${ }^{2}$ We have checked this for many different integer vectors $\mathbf{n}$ and parameters $\lambda$ in the case $q=0$ and $N=2$ using MAPLE.
} 
the functions $\mathcal{J}_{\mathbf{n}}(\mathbf{z})$ thus defined as an elliptic generalization of the Jack polynomials. It is important to note that the latter are no longer polynomials for $q>0$; see Remark 1 in Section 5 for a more precise characterization. Moreover, the corresponding eigenvalues $\mathcal{E}_{\mathbf{n}}$ and the coefficients $\alpha_{\mathbf{n}}(\mathbf{m})$ are more complicated [L1].

One main result in this paper is an implicit equation determining the eigenvalues $\mathcal{E}_{\mathbf{n}}$ and an explicit formula for the coefficients $\alpha_{\mathbf{n}}(\mathbf{m})$ depending on $\mathcal{E}_{\mathbf{n}}$ (Theorem 4.1.2). We also present a solution of these equations by some variant of perturbation theory to all orders. This leads to fully explicit formulas for $\mathcal{E}_{\mathbf{n}}$ and $\alpha_{\mathbf{n}}(\mathbf{m})$ as infinite series (Theorem 4.3.1). Our series are (essentially) power series in the parameter $\gamma$, but it is important to note that the small parameter is still $q^{2}$ and $\gamma$ is only a convenient book keeping device. A simple analogue illustrating this latter feature of our solution is the following function,

$$
f(q)=\sum_{s=2}^{\infty} \gamma^{s} \sum_{\nu_{1}, \ldots, \nu_{s} \in \mathbb{Z}} \delta\left(\nu_{1}+\nu_{2}+\cdots+\nu_{s}, 0\right) \prod_{\ell=1}^{s} \frac{\left|\nu_{\ell}\right| q^{2\left|\nu_{\ell}\right|}}{\left(1-q^{2\left|\nu_{\ell}\right|}\right)}
$$

with $\delta$ the Kronecker delta: this function has a complicated power series in $q^{2}$ while its power series $f(q)=\sum_{s=2}^{\infty} \gamma^{s} f_{s}\left(q^{2}\right)$ in $\gamma$ is simple and can be written explicitly. Moreover, it is not difficult to prove that $f_{s}=\mathcal{O}\left(q^{2\left\lceil\frac{s}{2}\right\rceil}\right)$ and that this series has a finite radius of convergence in $q^{2}$.

We also prove that our series for $\mathcal{E}_{\mathbf{n}}$ and $\alpha_{\mathbf{n}}(\mathbf{m})$ have a finite radius of convergence in $q^{2}$ under a certain hypothesis which is fulfilled in several interesting cases, including $N=2$ for non-integer $\lambda$ (Lamé case), and the groundstate $\mathbf{n}=\mathbf{0}$ for all $N>2$ and irrational $\lambda$. Our estimates to prove convergences are not optimal, and we believe that our series converge for all possible parameter values. In numerical computations it might be more efficient to solve the implicit equation for $\mathcal{E}_{\mathbf{n}}$ by iteration rather than using our explicit series.

\subsection{Previous results}

We now discuss previous results related to our work.

For $N=2$ the eigenvalue equation of the eCS differential operator can be reduced to the so-called Lamé equation which was studied extensively at the end of the $19^{\text {th }}$ century (see [WW] for a summary of these classical results) and more recently in [EK, R2]. It is known that, for integer values of $\lambda$, the Lamé equation has a finite number (depending on $\lambda$ ) of eigenfunctions and corresponding eigenvalues which can be computed algebraically [WW, and a generalization of this result to $N>2$ was found by Gómez-Ullate et.al [GGR] and led to particular many-body generalizations of the Lamé equation which, however, is different from the eCS model. There exist various other interesting results on the solution of the eCS model for integer values of $\lambda$ : Dittrich and Inozemtsev obtained explicit formulas for the eigenvalues of the eCS model for the cases $\lambda=2$ and $N=3$ [DI, I1] and $\lambda=2$ and general $N$ [12]. A Bethe ansatz solution of the integer- $\lambda$ eCS models and, more generally, the elliptic Ruijsenaars model [R1] (which reduces to the eCS model in a certain limit), was given by Felder and Varchenko [FV2, FV3]; see also [B, HSY, T]. There exists an interesting separation-of-variable approach to the solution of the $N=3$ eCS model by 
Sklyanin [S]. The latter approach seems to be similar to ours in that it is also based on an identity closely related to the one in Lemma 2.1.1, and it was elaborated in detail in the trigonometric limit $q=0$ by Kuznetsov et.al. [KMS]. We also mention the work of Núñez et.al [FGP] who computed the eigenvalues of the eCS model for arbitrary values of $\lambda$ up to $\mathcal{O}\left(q^{2}\right)$ for $N \leq 4$ and up to $\mathcal{O}\left(q^{4}\right)$ for $N=2$ and which our results extend to arbitrary orders and particle numbers. Other related work is by Komori and Takemura [KT] who presented a perturbative algorithm to solve the eCS model and proved that Schrödinger perturbation theory has a finite radius of convergence in $q^{2}$; see also [T]. As far as we can see is the approach in [KT] different from ours (we obtain results which are different from standard ones even for $q=0$ [L3]) and has not yielded results equally explicit as ours. A complimentary discussion of known results about eCS type systems and further references can be found in $[\mathrm{KR}]$.

As mentioned, our approach is based on a remarkable functional identity in Lemma 2.1.1. This identity can be regarded as a natural quantum analogue of the Bäcklund transformation discovered by Wojciechowski [W]; see also [KS]. Similar identities were obtained by Felder and Varchenko [FV1] and, for $N=3$, by Sklyanin [S]. Moreover, its trigonometric limit is equivalent to a well-known identity for the Jack polynomials due to Stanley; see Proposition 2.1 in Ref. [St]. Similar identities exist for a large class of Calogero-Sutherland type models and provide a tool to construct explicit formulas for the eigenfunctions of all these systems in a unified way [HL]. It is interesting to note that the functions $f_{\mathbf{n}}(\mathbf{z})$ for $q=0$ are the building blocks of this explicit solution for all these models.

The results presented in this paper evolved over several years. The key to our solution was found already in 2000 [L1], but we realized only in 2004 that it is possible to obtain an explicit solution to all orders [L5]. Readers interested in how this result emerged can consult the two earlier versions of the present paper [L4]. The first version ([L4] v1) is a manuscript from 2001 which remained unpublished until January 2004. It contains our solution algorithm together with a recipe how to compute the eigenvalues as power series in the nome $q$ of the elliptic functions. In the second version ([L4] v2) published in April 2004 we added explicit formulas for the eigenvalues up to $\mathcal{O}\left(q^{8}\right)$ for $N=2$ and up to $\mathcal{O}\left(q^{4}\right)$ for $N \leq 4$. These formulas were obtained by straightforward but tedious computations. These latter results suggested an alternative, more efficient, method allowing to find the solution to all orders in $q^{2}$. This result was previously announced in Refs. [L5, L7] and is elaborated and extended in the present paper.

A key issue in this evolution of our result was that we realized that there exist better answers to the question: "What parameter should we use to expand our solution in?", than the obvious one. The obvious answer is: "q" [L1]. However, this leads to very complicated formulas already at low orders. A better answer is: " $\gamma$ ", since it leads to much simpler formulas at low order; see Section [3. The final answer which allowed us to deduce explicit formulas to all orders is: " $\eta$ ", where $\eta$ an auxiliary parameter which we introduce into the problem and which can be set to 1 at the end of the computation. In particular, the explicit formulas for the eigenvalues which we thus obtain are of the form $\mathcal{E}_{\mathbf{n}}=\mathcal{E}_{0}(\mathbf{n})+\sum_{m=1}^{\infty} \eta^{m} \mathcal{E}_{m}(\mathbf{n})$ where $\mathcal{E}_{m}(\mathbf{n})=\mathcal{O}\left(q^{2 m}\right)$ are Taylor series in $q^{2}$; see Theorem 4.3.1 and Remark 4.3.2. 


\subsection{Plan of the rest of the paper}

In the next section we summarize preliminary results which we need. In Section 3 we derive the solution of the eCS model, i.e. its eigenfunctions and corresponding eigenvalues, as a series in $\gamma$ up to order $\mathcal{O}\left(\gamma^{3}\right)$ and $\mathcal{O}\left(\gamma^{4}\right)$, respectively, using an elementary argument. Our main results are obtained in Section 4: explicit formulas for the solution of the eCS model as a series in $\gamma$ to all orders, together with a sufficient condition for absolute convergence (Theorems 4.1.2 and 4.3.1). We end with remarks in Section 5, Details of our computations and some proofs are deferred to three appendices.

Notation: We denote as $\mathbb{Z}, \mathbb{Z}^{\prime}, \mathbb{N}_{0}$ and $\mathbb{N}$ the sets of all, all non-zero, all non-negative, and all positive integers, and $\mathbb{C}$ and $\mathbb{R}$ are the complex and real numbers, respectively. We use bold symbols for vectors with $N$ components, e.g. $\mathbf{n} \in \mathbb{Z}^{N}$ is short for $\left(n_{1}, \ldots, n_{N}\right)$ with $n_{j} \in \mathbb{Z}$ etc. The symbol " $\delta$ " always means the Kronecker delta, in particular, $\delta(\mathbf{m}, \mathbf{n})=$

$\prod_{j=1}^{N} \delta_{m_{j}, n_{j}}$ for $\mathbf{m}, \mathbf{n} \in \mathbb{Z}^{N}$. For $x \in \mathbb{R}$ we denote as $\lceil x\rceil$ the smallest integer larger or equal to $x$. Definitions are indicated by the symbol "def",

\section{Summary of preliminary results}

In this Section we collect a few results which we need.

\subsection{A remarkable identity}

The starting point for our solution method is the following.

Lemma 2.1.1 Let

$$
F(\mathbf{x} ; \mathbf{y}) \stackrel{\text { def }}{=} \frac{\prod_{1 \leq j<k \leq N} \theta\left(x_{k}-x_{j}\right)^{\lambda} \prod_{1 \leq j<k \leq N} \theta\left(y_{j}-y_{k}\right)^{\lambda}}{\prod_{j, k=1}^{N} \theta\left(x_{j}-y_{k}\right)^{\lambda}},
$$

with $\theta(r)$ in (1.5), where $\mathbf{x}=\left(x_{1}, \ldots, x_{N}\right) \in \mathbb{C}^{N}$ and similarly for $\mathbf{y}$. Then the following identity holds true,

$$
\sum_{j=1}^{N}\left(\frac{\partial^{2}}{\partial x_{j}^{2}}-\frac{\partial^{2}}{\partial y_{j}^{2}}\right) F(\mathbf{x} ; \mathbf{y})=2 \lambda(\lambda-1) \sum_{1 \leq j<k \leq N}\left(V\left(x_{k}-x_{j}\right)-V\left(y_{j}-y_{k}\right)\right) F(\mathbf{x} ; \mathbf{y})
$$

with $V(r)$ as in (1.6).

(Proof in Appendix A.3.)

As already mentioned, the result in Lemma 2.1.1 was obtain in [L2] using quantum field theory techniques. The proof given in Appendix A.3 is elementary and based on the following functional identity [WW]

$$
\phi(x) \phi(y)+\phi(x) \phi(z)+\phi(y) \phi(z)=f(x)+f(y)+f(z) \quad \text { if } x+y+z=0
$$


where

$$
\phi(x)=\frac{d}{d x} \log \theta(x), \quad f(x)=\frac{1}{2}\left[V(x)-\phi(x)^{2}-c_{0}\right]
$$

and $c_{0}$ the constant in (1.3); a proof of the latter identity is in Appendix A.2.

It is important to note that one can write the identity in (2.2) as follows,

$$
H(\mathbf{x}) F(\mathbf{x} ; \mathbf{y})=H(\mathbf{y}) F(\mathbf{x} ; \mathbf{y})
$$

where $H$ is the differential operator in (1.1) but acting on different arguments $\mathbf{x}$ and $\mathbf{y}$, as indicated.

\subsection{Reformulation of the eigenvalue problem}

From Lemma 2.1.1 a straightforward computation leads to a result which is the next step in our solution. To state this result we find it convenient to define

$$
\left(\mathbf{E}_{j k}\right)_{\ell} \stackrel{\text { def }}{=} \delta_{j \ell}-\delta_{k \ell} \quad \forall j, k, \ell=1, \ldots, N, \quad j<k
$$

and use the shorthand notation

$$
\sum_{\hat{\boldsymbol{\nu}}} S_{\nu} \alpha(\hat{\boldsymbol{\nu}}) \stackrel{\text { def }}{=} \sum_{1 \leq j<k \leq N} \sum_{\nu \in \mathbb{Z}} S_{\nu} \alpha\left(\nu \mathbf{E}_{j k}\right)
$$

for functions $\alpha$ on integer vectors in $\mathbb{Z}^{N}$.

Proposition 2.2.1 Let

$$
\hat{F}_{\mathbf{n}}(\mathbf{x}) \stackrel{\text { def }}{=} f_{\mathbf{n}}(\mathbf{z}) \Psi_{0}(\mathbf{x}), \quad \mathbf{n} \in \mathbb{Z}^{N} \text { and } z_{j}=\mathrm{e}^{\mathrm{i} x_{j}}
$$

with $\Psi_{0}(\mathbf{x})$ defined in (1.9) and (1.5) and the special functions

$$
f_{\mathbf{n}}(\mathbf{z}) \stackrel{\text { def }}{=}\left(\prod_{j=1}^{N} \oint_{\mathcal{C}_{j}} \frac{\mathrm{d} \xi_{j}}{2 \pi \mathrm{i} \xi_{j}} \xi_{j}^{n_{j}}\right) \frac{\prod_{1 \leq j<k \leq N} \Theta\left(\xi_{j} / \xi_{k}\right)^{\lambda}}{\prod_{j, k=1}^{N} \Theta\left(z_{j} / \xi_{k}\right)^{\lambda}}
$$

with the integration paths

$$
\mathcal{C}_{j}: \xi_{j}=\mathrm{e}^{\varepsilon_{j}} \mathrm{e}^{\mathrm{i} \varphi_{j}}, \quad-\pi \leq \varphi_{j} \leq \pi, \quad 0<\varepsilon_{1}<\varepsilon_{2}<\cdots<\varepsilon_{N}<\beta
$$

and

$$
\Theta(z) \stackrel{\text { def }}{=}(1-z) \prod_{m=1}^{\infty}\left[\left(1-q^{2 m} z\right)\left(1-q^{2 m} / z\right)\right]
$$

Then the eCS differential operator $H$ in (1.1)-(1.2) obeys

$$
H \hat{F}_{\mathbf{n}}(\mathbf{x})=\mathcal{E}_{0}(\mathbf{n}) \hat{F}_{\mathbf{n}}(\mathbf{x})-\gamma \sum_{\hat{\boldsymbol{\nu}}} S_{\nu} \hat{F}_{\mathbf{n}+\hat{\boldsymbol{\nu}}}(\mathbf{x})
$$


where

$$
\mathcal{E}_{0}(\mathbf{n}) \stackrel{\text { def }}{=} \sum_{j=1}^{N}\left(n_{j}+\frac{1}{2} \lambda(N+1-2 j)\right)^{2}
$$

and

$$
S_{\nu} \stackrel{\text { def }}{=}|\nu| \frac{q^{|\nu|-\nu}}{1-q^{2|\nu|}} \quad \forall \nu \in \mathbb{Z}^{\prime}, \quad S_{0} \stackrel{\text { def }}{=} 0 .
$$

(Proof in Appendix B.)

This result concluded our discussion in [L2] $3^{3}$ Our proof in Appendix B is (essentially) by expanding the identity in (2.5) in a Laurent series in the variables $\xi_{j}=\mathrm{e}^{\mathrm{i} y_{j}}$ in a certain region in $\mathbb{C}^{N}$ and equating the expansion coefficients.

As discussed below, the integrals in (2.9) -(2.11) are well-defined and independent of the parameters $\varepsilon_{j}$ in the specified range due to Cauchy's theorem. It is important to note that $S_{\nu}=0$ for $\nu \leq 0$ and $q=0$. As we will see, this simplifies the solution for $q=0$ drastically.

An important consequence of Proposition 2.2.1 is the following.

\section{Corollary 2.2.2 Let}

$$
\Psi(\mathbf{x})=\sum_{\mathbf{m} \in \mathbb{Z}^{N}} \alpha(\mathbf{m}) \hat{F}_{\mathbf{m}}(\mathbf{x})
$$

with coefficients $\alpha(\mathbf{m})$ satisfying the following relations,

$$
\left[\mathcal{E}_{0}(\mathbf{m})-\mathcal{E}\right] \alpha(\mathbf{m})=\gamma(\mathbb{S} \alpha)(\mathbf{m})
$$

for some constant $\mathcal{E}$ and

$$
(\mathbb{S} \alpha)(\mathbf{m}) \stackrel{\text { def }}{=} \sum_{\hat{\boldsymbol{\nu}}} S_{\nu} \alpha(\mathbf{m}-\hat{\boldsymbol{\nu}})
$$

with $\hat{F}_{\mathbf{n}}(\mathbf{x}), \mathcal{E}_{0}(\mathbf{n})$, and $S_{\nu}$ defined in Proposition 2.2.1 and $\hat{\boldsymbol{\nu}}$ in 2.7). Then $\Psi(\mathbf{x})$ is an eigenfunction of the eCS Hamiltonian in (1.1) with eigenvalue $\mathcal{E}: H \Psi(\mathbf{x})=\mathcal{E} \Psi(\mathbf{x})$.

This is a simple consequence of the ansatz (2.15) and Proposition 2.2.1 which imply

$$
(H-\mathcal{E}) \Psi=\sum_{\mathbf{m}}\left(\left[\mathcal{E}_{0}(\mathbf{m})-\mathcal{E}\right] \alpha(\mathbf{m})-\gamma(\mathbb{S} \alpha)(\mathbf{m})\right) \hat{F}_{\mathbf{m}}(\mathbf{x}) .
$$

Thus the problem of solving the eCS model is reduced to finding solutions $\mathcal{E}$ and $\alpha(\mathbf{m})$ of (2.16).

\subsection{Properties of the functions $f_{\mathbf{n}}(\mathbf{z})$}

The functions $f_{\mathbf{n}}(\mathbf{z})$ defined in (2.9) -(2.11) play an important role in our solution. To see that they are well-defined we note that

$$
0<\Theta(|z|) \leq|\Theta(z)| \leq \Theta(-|z|)<\infty \text { for } q^{2}<|z|<1
$$

\footnotetext{
${ }^{3}$ Note that $f_{\mathbf{n}}(\mathbf{z})$ here was denoted as $\mathcal{P}(\mathbf{n} ; \mathbf{x})$ in $[\mathrm{L} 2$.
} 
(see Appendix C.1), and thus the integrand in (2.9) is analytic in the region $1<\left|\xi_{1}\right|<$ $\left|\xi_{2}\right|<\cdots<\left|\xi_{N}\right|<q^{-2}$ if all $\left|z_{j}\right|=1$. Thus the integral in (2.9) are well-defined and independent of the closed integration paths $\mathcal{C}_{j}$ as long as they are within this region of analyticity (Cauchy's theorem), which is obviously the case for the ones in (2.10).

It is important to note that the $f_{\mathbf{n}}(\mathbf{z})$ are symmetric functions: $f_{\mathbf{n}}\left(z_{1}, z_{2}, \ldots, z_{N}\right)=$ $f_{\mathbf{n}}\left(z_{\pi(1)}, z_{\pi(2)}, \ldots, z_{\pi(N)}\right)$ for all $\pi \in S_{N}$. Moreover, they are polynomials for $q=0$ [L3]. As already mentioned, for $q=0$ the $f_{\mathbf{n}}(\mathbf{z})$ provide a basis in the space of symmetric polynomials if the $\mathbf{n}$ are restricted to partitions, even though they are, in general, non-zero even for non-partition integer vectors $\mathbf{n}[\mathrm{L3}, \mathrm{HL}$. We expect that similar results hold true also for $q \neq 0$.

We finally state an upper bound for the functions $f_{\mathbf{n}}(\mathbf{z})$ which we need to prove square integrability of our eigenfunctions:

Lemma 2.3.1 The functions $f_{\mathbf{n}}(\mathbf{z})$ defined in (2.9) obey

$$
\left|f_{\mathbf{n}}(\mathbf{z})\right|<C q^{\sum_{j}\left(\tilde{K}\left|n_{j}\right|-K j n_{j}\right)}, \quad\left|z_{j}\right|=1
$$

with the constants

$$
K \stackrel{\text { def }}{=} \frac{2}{N+b}, \quad \tilde{K} \stackrel{\text { def }}{=} \frac{b K}{1+2 b}, \quad C \stackrel{\text { def }}{=} \frac{\left[2 \Theta\left(-q^{2}\right)\right]^{N(N-1) \lambda / 2}}{\left[\left(1-q^{K-\tilde{K}}\right) \Theta\left(q^{2-2 b \tilde{K}}\right) /\left(1-q^{2-2 b \tilde{K}}\right)\right]^{N^{2} \lambda}}
$$

for arbitrary $b>0$.

(Proof in Appendix C.1.)

Note that $0<\tilde{K}<K / 2,2-2 b \tilde{K}>0$, and $C<\infty$. It is worth mentioning that these estimates are not optimal (and in fact meaningless for $q=0$ ), but they are good enough for our purposes.

\subsection{Lagrange's reversion theorem}

We finally recall a well-known result due to Lagrange which will play a key role in our solution:

Theorem 2.4.1 (A) Let $\varphi(z)$ and $g(z)$ be functions of the complex variable $z$ analytic on and inside a closed contour $\mathcal{C}$ surrounding a point $z=a$, and $\eta$ a complex number such that

$$
\sup _{z \in \mathcal{C}} \frac{|\eta \varphi(z)-a|}{|z-a|}<1
$$

Then the equation $z=\eta \varphi(z)$ has a unique solution $z=\xi$ inside of $\mathcal{C}, g(\xi)$ can be expanded as

$$
g(\xi)=g(a)+\sum_{m=1}^{\infty} \frac{\eta^{m}}{m !} \frac{\mathrm{d}^{m-1}}{\mathrm{~d} a^{m-1}}\left(\varphi(a)^{m} \frac{\mathrm{d}}{\mathrm{d} a} g(a)\right)
$$

and this series is absolutely convergent. 
(B) The result in (2.22) holds true in the sense of formal power series even for values of $\eta$ where (2.21) is not fulfilled.

Proof: Part (A) is equivalent to Lagrange's theorem as stated in [WW], Section 7.32. The result in $(\mathrm{B})$ is a simple implication.

Remark 2.4.2 We note that (2.22) does not rely on analyticity and applies to even more general situations where one knows that $\varphi(z)$ and $g(z)$ have formal power series in $(z-a)$ but has no information about convergence of these series. Indeed, inserting

$$
\varphi(z)=\sum_{n=0}^{\infty} \varphi_{n}(z-a)^{n}
$$

into the equation $z=\eta \varphi(z)$ and making an ansatz $\xi=a+\sum_{n=1}^{\infty} \xi_{k} \eta^{k}$ for its solution one can solve for the $\xi_{k}$ recursively and compute

$$
\xi=a+\eta \varphi_{0}+\eta^{2} \varphi_{0} \varphi_{1}+\eta^{3}\left(\varphi_{0}^{2} \varphi_{2}+\varphi_{0} \varphi_{1}^{2}\right)+\eta^{4}\left(\varphi_{0}^{3} \varphi_{3}+3 \varphi_{0}^{2} \varphi_{1} \varphi_{2}+\varphi_{0} \varphi_{1}^{3}\right)+\ldots
$$

Inserting this in $g(z)=\sum_{n=0}^{\infty} g_{n}(z-a)^{n}$ yields

$$
\begin{aligned}
g(\xi) & =g_{0}+\eta g_{1} \varphi_{0}+\eta^{2}\left(g_{2} \varphi_{0}^{2}+g_{1} \varphi_{0} \varphi_{1}\right)+\eta^{3}\left(g_{3} \varphi_{0}^{3}+2 g_{2} \varphi_{0}^{2} \varphi_{1}\right. \\
& \left.+g_{1}\left[\varphi_{0}^{2} \varphi_{2}+\varphi_{0} \varphi_{1}^{2}\right]\right)+\eta^{4}\left(g_{4} \varphi_{0}^{4}+3 g_{3} \varphi_{0}^{3} \varphi_{1}+g_{2}\left[2 \varphi_{0}^{3} \varphi_{2}+3 \varphi_{0}^{2} \varphi_{1}^{2}\right]\right. \\
& \left.+g_{1}\left[\varphi_{0}^{3} \varphi_{3}+3 \varphi_{0}^{2} \varphi_{1} \varphi_{2}+\varphi_{0} \varphi_{1}^{3}\right]\right)+\ldots
\end{aligned}
$$

which can be straightforwardly extended to higher powers in $\eta$ and agrees with (2.22) to all orders in $\eta$.

\section{Perturbative solution}

We will present our solution to all orders in the next section. As a heuristic motivation, we present in this section a pedestrian approach allowing to compute the first few terms of our series solution in a simple manner.

We found that (2.16) can be efficiently solved by making the ansatz

$$
\alpha(\mathbf{m})=\sum_{s=0}^{\infty} \gamma^{s} \alpha^{(s)}(\mathbf{m}), \quad \mathcal{E}=\sum_{s=0}^{\infty} \gamma^{s} \mathcal{E}^{(s)}
$$

leading to the following system of equations equivalent to (2.16),

$$
\left[\mathcal{E}_{0}(\mathbf{m})-\mathcal{E}^{(0)}\right] \alpha^{(0)}(\mathbf{m})=0
$$

and

$$
\left[\mathcal{E}_{0}(\mathbf{m})-\mathcal{E}^{(0)}\right] \alpha^{(s)}(\mathbf{m})=\sum_{s^{\prime}=1}^{s} \mathcal{E}^{\left(s^{\prime}\right)} \alpha^{\left(s-s^{\prime}\right)}(\mathbf{m})+\left(\mathbb{S} \alpha^{(s-1)}\right)(\mathbf{m}) \quad \forall s>0
$$


As mentioned in the introduction, $\gamma$ here is not assumed to be small but only serves as a convenient book keeping parameter to organize our solution. It is important to note that, for each $\mathbf{n} \in \mathbb{Z}^{N}$, the initial condition in (3.2) has the following solution,

$$
\mathcal{E}_{\mathbf{n}}^{(0)}=\mathcal{E}_{0}(\mathbf{n}), \quad \alpha_{\mathbf{n}}^{(0)}(\mathbf{m})=\delta_{\mathbf{n}}(\mathbf{m})
$$

where $\delta_{\mathbf{n}}(\mathbf{m}) \stackrel{\text { def }}{=} \delta(\mathbf{m}, \mathbf{n})$. Note that $\alpha_{\mathbf{n}}^{(s>0)}(\mathbf{n})$ is then undetermined by (3.3) and could be set to any value. This ambiguity corresponds to the freedom of multiplying the eigenfunction by an $\gamma$-dependent constant. Our choice below is a convenient normalization.

Equation (3.3) implies

$$
\mathcal{E}_{\mathbf{n}}^{(s)}=-\left(\mathbb{S} \alpha_{\mathbf{n}}^{(s-1)}\right)(\mathbf{n}) \quad \forall s>0
$$

where we set $\alpha_{\mathbf{n}}^{(s)}(\mathbf{n})=0$ for all $s>0$, and

$$
\alpha_{\mathbf{n}}^{(s)}(\mathbf{m})=\frac{1}{b_{\mathbf{n}}(\mathbf{m}-\mathbf{n})}\left(\sum_{s^{\prime}=1}^{s-1} \mathcal{E}_{\mathbf{n}}^{\left(s^{\prime}\right)} \alpha_{\mathbf{n}}^{\left(s-s^{\prime}\right)}(\mathbf{m})+\left(\mathbb{S} \alpha_{\mathbf{n}}^{(s-1)}\right)(\mathbf{m})\right) \quad \forall s>0 ;
$$

we use the convenient shorthand notation

$$
\frac{1}{b_{\mathbf{n}}(\mathbf{m}-\mathbf{n})} \stackrel{\text { def }}{=}\left\{\begin{array}{ll}
0 & \text { if } \mathbf{m}=\mathbf{n} \\
{\left[\mathcal{E}_{0}(\mathbf{m})-\mathcal{E}_{0}(\mathbf{n})\right]^{-1}} & \text { otherwise }
\end{array} .\right.
$$

We thus can compute, recursively, all $\mathcal{E}_{\mathbf{n}}^{(s)}$ and $\alpha_{\mathbf{n}}^{(s)}(\mathbf{m})$. We obtain

$$
\begin{aligned}
& \mathcal{E}_{\mathbf{n}}^{(1)}=-\sum_{\hat{\boldsymbol{\nu}} \in \mathbb{Z}} S_{\nu} \delta(\mathbf{0}, \hat{\boldsymbol{\nu}})=0, \\
& \alpha_{\mathbf{n}}^{(1)}(\mathbf{m})=\sum_{\hat{\boldsymbol{\nu}} \in \mathbb{Z}} S_{\nu} \frac{\delta(\mathbf{m}-\mathbf{n}, \hat{\boldsymbol{\nu}})}{b_{\mathbf{n}}(\hat{\boldsymbol{\nu}})}, \\
& \mathcal{E}_{\mathbf{n}}^{(2)}=-\sum_{\hat{\boldsymbol{\nu}}_{1}, \hat{\boldsymbol{\nu}}_{2}} S_{\nu_{1}} S_{\nu_{2}} \frac{\delta\left(\mathbf{0}, \hat{\boldsymbol{\nu}}_{1}+\hat{\boldsymbol{\nu}}_{2}\right)}{b_{\mathbf{n}}\left(\hat{\boldsymbol{\nu}}_{1}\right)}=-\sum_{\hat{\boldsymbol{\nu}} \in \mathbb{Z}} S_{\nu} S_{-\nu} \frac{1}{b_{\mathbf{n}}(\hat{\boldsymbol{\nu}})}, \\
& \alpha_{\mathbf{n}}^{(2)}(\mathbf{m})=\sum_{\hat{\boldsymbol{\nu}}_{1}, \hat{\boldsymbol{\nu}}_{2}} S_{\nu_{1}} S_{\nu_{2}} \frac{\delta\left(\mathbf{m}-\mathbf{n}, \hat{\boldsymbol{\nu}}_{1}+\hat{\boldsymbol{\nu}}_{2}\right)}{b_{\mathbf{n}}\left(\hat{\boldsymbol{\nu}}_{1}+\hat{\boldsymbol{\nu}}_{2}\right) b_{\mathbf{n}}\left(\hat{\boldsymbol{\nu}}_{1}\right)} \\
& \mathcal{E}_{\mathbf{n}}^{(3)}=-\sum_{\hat{\boldsymbol{\nu}}_{1}, \hat{\boldsymbol{\nu}}_{2}, \hat{\boldsymbol{\nu}}_{3}} S_{\nu_{1}} S_{\nu_{2}} S_{\nu_{3}} \frac{\delta\left(\mathbf{0}, \hat{\boldsymbol{\nu}}_{1}+\hat{\boldsymbol{\nu}}_{2}+\hat{\boldsymbol{\nu}}_{3}\right)}{b_{\mathbf{n}}\left(\hat{\boldsymbol{\nu}}_{1}+\hat{\boldsymbol{\nu}}_{2}\right) b_{\mathbf{n}}\left(\hat{\boldsymbol{\nu}}_{1}\right)} \\
& \alpha_{\mathbf{n}}^{(3)}(\mathbf{m})=\sum_{\hat{\boldsymbol{\nu}}_{1}, \hat{\boldsymbol{\nu}}_{2}, \hat{\boldsymbol{\nu}}_{3}} S_{\nu_{1}} S_{\nu_{2}} S_{\nu_{3}}\left(-\frac{\delta\left(\mathbf{0}, \hat{\boldsymbol{\nu}}_{1}+\hat{\boldsymbol{\nu}}_{2}\right) \delta\left(\mathbf{m}-\mathbf{n}, \hat{\boldsymbol{\nu}}_{3}\right)}{b_{\mathbf{n}}\left(\hat{\boldsymbol{\nu}}_{1}\right) b_{\mathbf{n}}\left(\hat{\boldsymbol{\nu}}_{3}\right)^{2}}+\right. \\
& \left.\frac{\delta\left(\mathbf{m}-\mathbf{n}, \hat{\boldsymbol{\nu}}_{1}+\hat{\boldsymbol{\nu}}_{2}+\hat{\boldsymbol{\nu}}_{3}\right)}{b_{\mathbf{n}}\left(\hat{\boldsymbol{\nu}}_{1}+\hat{\boldsymbol{\nu}}_{2}+\hat{\boldsymbol{\nu}}_{3}\right) b_{\mathbf{n}}\left(\hat{\boldsymbol{\nu}}_{1}+\hat{\boldsymbol{\nu}}_{2}\right) b_{\mathbf{n}}\left(\hat{\boldsymbol{\nu}}_{1}\right)}\right) \\
& \mathcal{E}_{\mathbf{n}}^{(4)}=\sum_{\hat{\boldsymbol{\nu}}_{1}, \hat{\boldsymbol{\nu}}_{2}, \hat{\boldsymbol{\nu}}_{3}} S_{\nu_{1}} S_{\nu_{2}} S_{\nu_{3}} S_{\nu_{4}}\left(\frac{\delta\left(\mathbf{0}, \hat{\boldsymbol{\nu}}_{1}+\hat{\boldsymbol{\nu}}_{2}\right) \delta\left(\mathbf{0}, \hat{\boldsymbol{\nu}}_{3}+\hat{\boldsymbol{\nu}}_{4}\right)}{b_{\mathbf{n}}\left(\hat{\boldsymbol{\nu}}_{1}\right) b_{\mathbf{n}}\left(\hat{\boldsymbol{\nu}}_{3}\right)^{2}}-\right. \\
& \left.\frac{\delta\left(\mathbf{0}, \hat{\boldsymbol{\nu}}_{1}+\hat{\boldsymbol{\nu}}_{2}+\hat{\boldsymbol{\nu}}_{3}+\hat{\boldsymbol{\nu}}_{4}\right)}{b_{\mathbf{n}}\left(\hat{\boldsymbol{\nu}}_{1}+\hat{\boldsymbol{\nu}}_{2}+\hat{\boldsymbol{\nu}}_{3}\right) b_{\mathbf{n}}\left(\hat{\boldsymbol{\nu}}_{1}+\hat{\boldsymbol{\nu}}_{2}\right) b_{\mathbf{n}}\left(\hat{\boldsymbol{\nu}}_{1}\right)}\right)
\end{aligned}
$$


etc. It is straightforward but tedious to continue this computation to higher orders in $\gamma$. Below we present a more efficient computation method allowing us to derive closed formulas for $\mathcal{E}_{\mathbf{n}}$ and $\alpha_{\mathbf{n}}(\mathbf{m})$ to all orders in $\gamma$.

It is straightforward to expand $\mathcal{E}_{\mathbf{n}}^{(s)}$ and $\alpha_{\mathbf{n}}^{(s)}$ in powers of $q^{2}$, but the resulting formulas are rather complicated, and we therefore do not present them here 4

We thus find, for each $\mathbf{n} \in \mathbb{Z}^{N}$, an eigenfunction $\Psi_{\mathbf{n}}(\mathbf{x})$ and a corresponding eigenvalue $\mathcal{E}_{\mathbf{n}}$ as a formal power series in $\gamma$. However, it is important to note the following potential problem: It is possible that $\mathcal{E}_{0}(\mathbf{m})-\mathcal{E}_{0}(\mathbf{n})$ vanishes for some $\mathbf{m} \neq \mathbf{n}$ with $\sum_{j}\left(m_{j}-n_{j}\right)=0$ which appear in the above sums (recall that these series are built of terms $b_{\mathbf{n}}(\mathbf{m}-\mathbf{n})$ in (3.7) with $\mathbf{m}=\mathbf{n}+\sum_{\kappa=1}^{s} \hat{\boldsymbol{\nu}}_{\kappa} \neq \mathbf{n}$, and all such $\mathbf{m}$ obey this latter condition), and in this case the sums are not defined. We refer to such $\mathbf{m}$ as resonances. Thus the results in the present section make sense only if there are no resonances, i.e. if

$$
\mathcal{E}_{0}(\mathbf{m}) \neq \mathcal{E}_{0}(\mathbf{n}) \quad \forall \mathbf{m} \neq \mathbf{n} \text { with } \sum_{j=1}^{N}\left(m_{j}-n_{j}\right)=0
$$

Since there exist parameters $\lambda$ and $\mathbf{n}$ where resonances exist this is an important restriction.

We now discuss cases with resonances. For example, for $N=2$ we have

$$
\mathcal{E}_{0}(\mathbf{m})-\mathcal{E}_{0}(\mathbf{n})=2 \nu\left(\nu+n_{1}-n_{2}+\lambda\right), \quad \nu \stackrel{\text { def }}{=} m_{1}-n_{1}=-\left(m_{2}-n_{2}\right) \quad(N=2),
$$

and thus there is a resonance for $\nu=n_{2}-n_{1}-\lambda$ provided that $\lambda$ is an integer. It is easy to see that there exist resonances for all $\mathbf{n}$ and $N$ if $\lambda$ is an integer, but for $N>2$ there exist even resonances for non-integer $\lambda$. For example, for $N=3$ and $\mathbf{n}$ such that $\nu \stackrel{\text { def }}{=}\left(n_{1}-2 n_{2}+n_{3}\right) / 3$ is a non-zero integer, there is a resonance at $\mathbf{m}=\left(n_{1}-\nu, n_{2}+2 \nu, n_{3}-\nu\right)$ for arbitrary values of $\lambda$. Such $\lambda$-independent resonances exist for all $N>2$. It is also interesting to note that, if $N>2$, there exist infinitely many ways to represent a resonance as $\mathbf{m}=\mathbf{n}+\sum_{\kappa=1}^{m} \hat{\boldsymbol{\nu}}_{\kappa}$ for $m>2$ : this is true since

$$
\mathbf{E}_{j_{1} j_{2}}+\mathbf{E}_{j_{2} j_{3}}+\ldots+\mathbf{E}_{j_{l-1} j_{l}}-\mathbf{E}_{j_{1} j_{l}}=\mathbf{0} \quad \text { if } 1 \leq j_{1}<j_{2}<\cdots<j_{l} \leq N, \quad l \leq N .
$$

Due to this identity resonance can occur in the above sums infinitely many times.

However, it is important to note that there exist cases without resonances where the results of the present section apply. In particular, (3.10) shows that no resonance exists for $N=2$ and non-integer $\lambda$. Moreover, results from numerical experiments performed with MAPLE suggest that, for arbitrary particle numbers and non-integer $\lambda$, there exist infinitely many $\mathbf{n}$ without resonances.

Anyway, we show in the next section that this potential problem of resonances can be circumvented by resummations.

\section{Solution to all orders}

We now introduce some notation which allows us to extend the solution above to all orders.

\footnotetext{
${ }^{4}$ The interested reader can find a few such formulas [L4] v2.
} 


\subsection{Implicit solution}

We write (2.16) as

$$
(\mathbb{A}-\mathcal{E}) \alpha=\gamma \mathbb{S} \alpha
$$

with

$$
(\mathbb{A} \alpha)(\mathbf{m}) \stackrel{\text { def }}{=} \mathcal{E}_{0}(\mathbf{m}) \alpha(\mathbf{m}) .
$$

It is natural to interpret $\mathbb{A}$ and $\mathbb{S}$ as linear operators on the vector space $\operatorname{Map}\left(\mathbb{Z}^{N} ; \mathbb{C}\right)$ of functions $\alpha: \mathbb{Z}^{N} \rightarrow \mathbb{C}, \mathbf{m} \mapsto \alpha(\mathbf{m})$. (The topology of this space plays no role in our discussion and is therefore ignored.) It is also convenient to introduce the following projection on $\operatorname{Map}\left(\mathbb{Z}^{N} ; \mathbb{C}\right)$,

$$
\left(\mathbb{P}_{\mathbf{n}} \alpha\right)(\mathbf{m}) \stackrel{\text { def }}{=} \delta(\mathbf{m}, \mathbf{n}) \alpha(\mathbf{m}) .
$$

The equation in (4.1) can be solved using the following simple but powerful result.

Lemma 4.1.1 Let $\mathbb{A}$ and $\mathbb{B}$ be linear operators on a vector space $V$ and consider the eigenvalue equation

$$
(\mathbb{A}-\mathcal{E}) \alpha=\mathbb{B} \alpha
$$

with the eigenvalue $\mathcal{E}$. Let $\mathbb{P}$ be some projection on $V$ commuting with $\mathbb{A}$ and $\mathbb{P}^{\perp} \stackrel{\text { def }}{=} I-\mathbb{P}$. Then

$$
\alpha=\left[I+\left(\mathbb{A}-\mathcal{E}-\mathbb{P}^{\perp} \mathbb{B}\right)^{-1} \mathbb{P}^{\perp} \mathbb{B}\right] \alpha_{0}
$$

is a solution of this eigenvalue equation provided that $\mathcal{E}$ and $\alpha_{0}$ satisfy the following conditions,

$$
\mathbb{P} \alpha_{0}=\alpha_{0}
$$

and

$$
\mathcal{E} \alpha_{0}=\mathbb{A} \alpha_{0}-\mathbb{P B}\left[I+\left(\mathbb{A}-\mathcal{E}-\mathbb{P}^{\perp} \mathbb{B}\right)^{-1} \mathbb{P}^{\perp} \mathbb{B}\right] \alpha_{0}
$$

Proof: Applying the projections $\mathbb{P}$ to (4.4) and inserting

$$
\alpha=\alpha_{0}+\mathbb{P}^{\perp} \alpha, \quad \alpha_{0} \stackrel{\text { def }}{=} \mathbb{P} \alpha
$$

we obtain

$$
\mathcal{E} \alpha_{0}=\mathbb{A} \alpha_{0}-\mathbb{P B} \alpha
$$

where we used that $\mathbb{A}$ and $\mathbb{P}$ commute. In a similar manner, applying $\mathbb{P}^{\perp}$ to (4.4), we get

$$
\mathbb{A P}^{\perp} \alpha-\mathcal{E} \mathbb{P}^{\perp} \alpha-\mathbb{P}^{\perp} \mathbb{B P}^{\perp} \alpha=\mathbb{P}^{\perp} \mathbb{B} \alpha_{0}
$$

Obviously the last two equations together are equivalent to (4.4). From the last equation we get

$$
\mathbb{P}^{\perp} \alpha=\left(\mathbb{A}-\mathcal{E}-\mathbb{P}^{\perp} \mathbb{B}\right)^{-1} \mathbb{P}^{\perp} \mathbb{B} \alpha_{0}
$$

which implies (4.5). Inserting (4.5) in (4.8) we obtain the condition in (4.7).

If we apply this result to (4.1) using the projection $\mathbb{P}=\mathbb{P}_{\mathbf{n}}$ and $\mathbb{B}=\gamma \mathbb{S}$ we can compute a solution $\alpha=\alpha_{\mathbf{n}}$ and $\mathcal{E}=\mathcal{E}_{\mathbf{n}}$. It is easy to solve (4.6): its general solution is $\alpha_{0}=c \delta_{\mathbf{n}}$ 
with $\delta_{\mathbf{n}}(\mathbf{m})=\delta(\mathbf{n}, \mathbf{m})$ and an arbitrary constant $c$. It is obvious that $c$ is an overall normalization constant which can be set to 1 without loss of generality. This is equivalent to our normalization condition $\alpha_{\mathbf{n}}^{(0)}(\mathbf{n})=1$ and $\alpha_{\mathbf{n}}^{(s)}=0$ for $s>1$ in the previous section. We thus obtain

$$
\alpha_{\mathbf{n}}=\left[I+\gamma\left(\mathbb{A}-\mathcal{E}-\gamma \mathbb{P}^{\perp} \mathbb{S}\right)^{-1} \mathbb{P}^{\perp} \mathbb{S}\right] \delta_{\mathbf{n}}
$$

and the condition in (4.8) determining the eigenvalue becomes

$$
\mathcal{E}_{\mathbf{n}}=\mathcal{E}_{0}(\mathbf{n})-\gamma\left(\mathbb{S} \alpha_{\mathbf{n}}\right)(\mathbf{n})
$$

We expand $\alpha_{\mathbf{n}}$ in a geometric series

$$
\alpha_{\mathbf{n}}=\sum_{s=0}^{\infty}\left(\gamma(\mathbb{A}-\mathcal{E})^{-1} \mathbb{P}^{\perp} \mathbb{S}\right)^{s} \delta_{\mathbf{n}}
$$

where we introduce the convenient notation

$$
\frac{1}{\left[\left[\mathcal{E}_{0}(\mathbf{m})-\mathcal{E}\right]\right]_{\mathbf{n}}} \stackrel{\text { def }}{=} \begin{cases}0 & \text { if } \mathbf{m}=\mathbf{n} \\ {\left[\mathcal{E}_{0}(\mathbf{m})-\mathcal{E}\right]^{-1}} & \text { otherwise }\end{cases}
$$

which allows us to write

$$
\left([\mathbb{A}-\mathcal{E}]^{-1} \mathbb{P}^{\perp} \alpha\right)(\mathbf{m})=\frac{1}{\left[\left[\mathcal{E}_{0}(\mathbf{m})-\mathcal{E}\right]\right]_{\mathbf{n}}} \alpha(\mathbf{m}) .
$$

We thus obtain our first main result.

Theorem 4.1.2 Let $\mathbf{n} \in \mathbb{Z}^{N}$ and

$$
\Psi_{\mathbf{n}}(\mathbf{x})=\sum_{\mathbf{m} \in \mathbb{Z}^{N}} \alpha_{\mathbf{n}}(\mathbf{m}) \hat{F}_{\mathbf{m}}(\mathbf{x})
$$

where

$$
\alpha_{\mathbf{n}}(\mathbf{m})=\delta(\mathbf{n}, \mathbf{m})+\sum_{s=1}^{\infty} \gamma^{s} \sum_{\hat{\boldsymbol{\nu}}_{1}, \ldots, \hat{\boldsymbol{\nu}}_{s}} \prod_{\kappa=1}^{s} S_{\nu_{\kappa}} \frac{\delta\left(\mathbf{m}, \mathbf{n}+\sum_{\kappa=1}^{s} \hat{\boldsymbol{\nu}}_{\kappa}\right)}{\prod_{\kappa=1}^{s}\left[\left[\mathcal{E}_{0}\left(\mathbf{n}+\sum_{\ell=1}^{\kappa} \hat{\boldsymbol{\nu}}_{\ell}\right)-\mathcal{E}_{\mathbf{n}}\right]\right]_{\mathbf{n}}}
$$

with $\hat{F}_{\mathbf{n}}(\mathbf{x}), \mathcal{E}_{0}(\mathbf{n}), S_{\nu}, \hat{\boldsymbol{\nu}}$ defined in Proposition 2.2.1, and $\mathcal{E}_{\mathbf{n}}$ a solution of the following equation,

$$
\mathcal{E}_{\mathbf{n}}=\mathcal{E}_{0}(\mathbf{n})-\sum_{s=2}^{\infty} \gamma^{s} \sum_{\hat{\boldsymbol{\nu}}_{1}, \ldots, \hat{\boldsymbol{\nu}}_{s}} \prod_{\kappa=1}^{s} S_{\nu_{\kappa}} \frac{\delta\left(\mathbf{0}, \sum_{\kappa=1}^{s} \hat{\boldsymbol{\nu}}_{\kappa}\right)}{\prod_{\kappa=1}^{s-1}\left[\left[\mathcal{E}_{0}\left(\mathbf{n}+\sum_{\ell=1}^{\kappa} \hat{\boldsymbol{\nu}}_{\ell}\right)-\mathcal{E}_{\mathbf{n}}\right]\right]_{\mathbf{n}}}
$$

Then $\Psi_{\mathbf{n}}(\mathbf{x})$ is an eigenfunction of the eCS differential operator $H$ in (1.1)-(1.2) with eigenvalue $\mathcal{E}_{\mathbf{n}}: H \Psi_{\mathbf{n}}(\mathbf{x})=\mathcal{E}_{\mathbf{n}} \Psi_{\mathbf{n}}(\mathbf{x})$. 
Our arguments above prove that this result holds true at least in the sense of formal power series in the nome $q$ of the elliptic functions, but, as we discuss in the next section, in many cases the series have a finite radius of convergence.

As before can the sum in (4.10) be restricted to $\mathbf{m} \in \mathbb{Z}$ satisfying $\sum_{j=1}^{N}\left(m_{j}-n_{j}\right)=0$.

Note that (4.12) is an implicit equation determining the eigenvalues of the eCS model. It is straightforward to obtain from it a solution as a formal power series in $\gamma$ and thus recover the results in the previous section. It is important to note that the problem of resonances has disappeared in (4.11) and (4.12), and it only reemerges if one solves these equations by a particular series expansion (which is essentially the one discussed in the previous section). However, it is possible to compute other series solutions avoiding resonances.

Remark 4.1.3 It is important to note that (4.12) simplifies in the trigonometric limit as follows,

$$
\mathcal{E}_{\mathbf{n}}=\mathcal{E}_{0}(\mathbf{n}) \text { for } q=0
$$

(since for $q=0, S_{\nu}$ is non-zero only for $\nu>0$, but all the Kronecker deltas in the sums on the r.h.s. of (4.12) are zero if all $\nu_{r}>0$ ), and we thus recover the well-known eigenvalues of the Sutherland model [Su1]. Thus in this case, Theorem 4.1.2 provides fully explicit and well-defined eigenfunctions $\Psi_{\mathbf{n}}(\mathbf{x})$ : one can prove that for all partitions $\mathbf{n}, \mathcal{E}_{0}\left(\mathbf{n}+\sum_{\ell=0}^{\kappa} \hat{\boldsymbol{\nu}}_{\ell}\right)-$ $\mathcal{E}_{0}(\mathbf{n})>0$ for all $\kappa>0$ and $\nu_{\ell}>0$ [L3], and thus resonances do not appear in [4.11] for $q=0$. As already mentioned, for partitions $\mathbf{n}$ and for $q=0$ these eigenfunctions are the same as Sutherland's [L3, HL]. In the following we assume $q>0$.

We now introduce some useful notation. We write (4.12) as follows,

$$
\tilde{\mathcal{E}}_{\mathbf{n}}=\Phi_{\mathbf{n}}\left(\tilde{\mathcal{E}}_{\mathbf{n}}\right) \text { where } \tilde{\mathcal{E}}_{\mathbf{n}} \stackrel{\text { def }}{=} \mathcal{E}_{\mathbf{n}}-\mathcal{E}_{0}(\mathbf{n})
$$

and

$$
\Phi_{\mathbf{n}}(z) \stackrel{\text { def }}{=}-\sum_{s=2}^{\infty} \gamma^{s} \sum_{\hat{\boldsymbol{\nu}}_{1}, \ldots, \hat{\boldsymbol{\nu}}_{s}} \prod_{\kappa=1}^{s} S_{\nu_{\kappa}} \frac{\delta\left(\mathbf{0}, \sum_{\kappa=1}^{s} \hat{\boldsymbol{\nu}}_{\kappa}\right)}{\prod_{\kappa=1}^{s-1}\left[\left[\mathcal{E}_{0}\left(\mathbf{n}+\sum_{\ell=1}^{\kappa} \hat{\boldsymbol{\nu}}_{\ell}\right)-\mathcal{E}_{0}(\mathbf{n})-z\right]\right]_{\mathbf{n}}}
$$

is a complex valued function of one complex variable $z$. Similarly we write (4.11) as

$$
\alpha_{\mathbf{n}}(\mathbf{m})=G_{\mathbf{n}}\left(\tilde{\mathcal{E}}_{\mathbf{n}} ; \mathbf{m}\right)
$$

where

$$
G_{\mathbf{n}}(z ; \mathbf{m}) \stackrel{\text { def }}{=} \delta(\mathbf{n}, \mathbf{m})+\sum_{s=1}^{\infty} \gamma^{s} \sum_{\hat{\boldsymbol{\nu}}_{1}, \ldots, \hat{\boldsymbol{\nu}}_{s}} \prod_{\kappa=1}^{s} S_{\nu_{\kappa}} \frac{\delta\left(\mathbf{m}, \mathbf{n}+\sum_{\kappa=1}^{s} \hat{\boldsymbol{\nu}}_{\kappa}\right)}{\prod_{\kappa=1}^{s}\left[\left[\mathcal{E}_{0}\left(\mathbf{n}+\sum_{\ell=1}^{\kappa} \hat{\boldsymbol{\nu}}_{\ell}\right)-\mathcal{E}_{0}(\mathbf{n})-z\right]\right]_{\mathbf{n}}}
$$

(for fixed $\mathbf{n}$ and $\mathbf{m}$ ) is also a complex function. 


\subsection{Analyticity results}

In this subsection we establish results concerning the analyticity of the functions $\Phi_{\mathbf{n}}(z)$ and $G_{\mathbf{n}}(z ; \mathbf{m})$ introduced at the end of the previous section. This is rather technical but needed to prove convergence of our series solution.

As we show below, for sufficiently small values of $q$, the above mentioned functions are analytic in certain regions of the complex $z$-plane. To simplify our discussion we now state a certain non-degeneracy condition which, when fulfilled, allows us to give a simple proof of convergence. We emphasis that neither this hypothesis nor the estimates we deduce from it are optimal, which is why we only get a proof of convergence in certain special cases.

Hypothesis 4.2.1 The model parameters $N, \lambda$ and $\mathbf{n} \in \mathbb{Z}^{N}$ are such that there exist constants $a \in \mathbb{R}$ and $\Delta>|a|$ satisfying

$$
\left|\mathcal{E}_{0}(\mathbf{m})-\mathcal{E}_{0}(\mathbf{n})-a\right| \geq \Delta>0 \quad \forall \mathbf{m} \neq \mathbf{n} \quad \text { such that } \sum_{j=1}^{N}\left(m_{j}-n_{j}\right)=0 .
$$

Note that $a$ and $\Delta$ can depend on $\mathbf{n}, N$ and $\lambda$.

It is interesting to note that, for rational values of $\lambda$, it is trivial to find constants $a \neq 0$ and $\Delta \geq|a|$ such that the condition in (4.18) is fulfilled:

Lemma 4.2.2 Let $\lambda$ be rational and $m$ and $n>0$ co-prime integers such that $\lambda=n / m$. Then any of the following parameters

$$
a=k_{1}+\lambda k_{2}+a_{0}, \quad k_{1}, k_{2} \in \mathbb{Z} \text { and } \Delta=\left|a_{0}\right| \leq \frac{1}{2 m}
$$

obey the condition in 4.18).

Proof: Equation (2.13) implies that $\mathcal{E}_{0}(\mathbf{n})-\mathcal{E}_{0}(\mathbf{m})$ is always of the form $\nu_{1}+\lambda \nu_{2}$ for some integers $\nu_{1,2}$, and thus it is obvious that $\left|\nu_{1}+\lambda \nu_{2}-a\right| \geq \Delta$ for all integers $\nu_{1,2}$ and the parameters in (4.19).

This lemma is enough to establish that the functions defined at the end of Section 4.1 are analytic in non-trivial $z$-regions for sufficiently small values of $q$ (Proposition 4.2.3). However, to prove that the prerequisites for Lagrange's theorem are fulfilled (Corollary 4.2.4) we need that $\Delta$ is strictly larger than $|a|$, and it is this which is non-trivial in our hypothesis. At the end of this section we prove that Hypothesis 4.2.1 is fulfilled for all $\mathbf{n}$ if $N=2$ and $\lambda \neq \mathbb{N}$ (Lemma 4.2.6). For $N>2$ we only have some partial results, including the groundstate $\mathbf{n}=\mathbf{0}$ and irrational $\lambda$ (Lemma 4.2.7).

We now are ready to state our analyticity result:

Proposition 4.2.3 Let $a \in \mathbb{R}$ and $\Delta>0$ be such that the condition in (4.18) holds true, $b \geq 0$ a parameter which can be chosen arbitrarily, and $q$ sufficiently small so that

$$
B \stackrel{\text { def }}{=} \frac{N(N-1)|\gamma| q^{2 /(N+b)}}{\left(1-q^{2 /(N+b)}\right)^{3}}
$$


satisfies $B<\Delta$. Then $\Phi_{\mathbf{n}}(z)$ in (4.15) and $G_{\mathbf{n}}(z ; \mathbf{m})$ in 4.17$)$ are analytic functions in the following region

$$
|z-a|<\Delta-B
$$

of the complex z-plane. Moreover, in that region the following estimates hold true,

$$
\left|\Phi_{\mathbf{n}}(z)\right|<\frac{B^{2}}{\Delta-B-|z-a|}
$$

and

$$
\left|G_{\mathbf{n}}(z ; \mathbf{m})\right|<\delta(\mathbf{m}, \mathbf{n})+q^{\sum_{j} K j\left(m_{j}-n_{j}\right)} \frac{B}{\Delta-B-|z-a|}
$$

with the constant $K$ in (2.20).

Proof: The estimate in (4.18) implies

$$
\left|\frac{1}{\mathcal{E}_{0}(\mathbf{m})-\mathcal{E}_{0}(\mathbf{n})-z}\right| \leq \frac{1}{\left|\mathcal{E}_{0}(\mathbf{m})-\mathcal{E}_{0}(\mathbf{n})-a\right|-|z-a|} \leq \frac{1}{\Delta-|z-a|}
$$

for all $\mathbf{m}=\mathbf{n}+\sum_{\ell} \hat{\boldsymbol{\nu}}_{\ell} \neq \mathbf{n}$ and $|z-a|<\Delta$, and thus (4.15) yields

$$
\left|\Phi_{\mathbf{n}}(z)\right| \leq \sum_{s=2}^{\infty}\left(\frac{1}{\Delta-|z-a|}\right)^{s-1} K_{s}(\mathbf{0})
$$

where

$$
K_{s}(\mathbf{m}) \stackrel{\text { def }}{=}|\gamma|^{s} \sum_{\hat{\boldsymbol{\nu}}_{1}, \ldots, \hat{\boldsymbol{\nu}}_{s}} \prod_{\kappa=1}^{s} S_{\nu_{\kappa}} \delta\left(\mathbf{m}, \sum_{\kappa=1}^{s} \hat{\boldsymbol{\nu}}_{\kappa}\right)>0
$$

for all $\mathbf{m} \in \mathbb{Z}^{N}$ and $s \in \mathbb{N}$; we used $S_{\nu} \geq 0$. One can prove that

$$
K_{s}(\mathbf{m}) \leq q^{2 \sum_{j} j m_{j} /(N+b)} B^{s}
$$

with $B$ in (4.20) and $b>0$ arbitrary; see Appendix C.2 for details. This implies

$$
\left|\Phi_{\mathbf{n}}(z)\right| \leq B \sum_{s=2}^{\infty}\left(\frac{B}{\Delta-|z-a|}\right)^{s-1}
$$

provided that $|z-a|<\Delta-B$. Summing up the geometric series we obtain the estimate in (4.22). It is obvious that each term in the series defining $\Phi_{\mathbf{n}}(z)$ in (4.15) is an analytical function in the region $|z-a|<\Delta$, and thus our estimates above prove that $\Phi_{\mathbf{n}}(z)$ converges and defines an analytic function in the region defined in (4.21); see e.g. [WW], Section 5.3.

The proof of analyticity of $G_{\mathbf{n}}(z ; \mathbf{m})$ in (4.17) is similar: we now can estimate

$$
\left|G_{\mathbf{n}}(z ; \mathbf{m})\right| \leq \delta(\mathbf{n}, \mathbf{m})+\sum_{s=1}^{\infty}\left(\frac{1}{\Delta-|z-a|}\right)^{s} K_{s}(\mathbf{m}-\mathbf{n})
$$

with $K_{s}(\mathbf{m})$ in (4.24). Using the estimate in (4.25) we obtain

$$
\left|G_{\mathbf{n}}(z ; \mathbf{m})\right| \leq \delta(\mathbf{n}, \mathbf{m})+\sum_{s=1}^{\infty} q^{2 \sum_{j} j\left(m_{j}-n_{j}\right) /(N+b)}\left(\frac{B}{\Delta-|z-a|}\right)^{s}
$$


which implies the estimate in (4.23) provided that $|z-a|<\Delta-B$. Similarly as above we conclude that $G_{\mathbf{n}}(z ; \mathbf{m})$ is analytic in the region defined in (4.21).

We solve the equation in (4.14) by using Lagrange's theorem 2.4.1. The following result gives a sufficient condition that the prerequisites for the stronger version (A) of this theorem are fulfilled:

Corollary 4.2.4 Let $a \in \mathbb{R}$ and $\Delta>|a|$ be such that the condition in (4.18) holds true, $b>0$ an arbitrary parameter, and $q$ sufficiently small so that $B$ in (4.20) satisfies $B<$ $(\Delta-|a|) / 3$. Then for any of the following closed contours in the complex z-plane,

$$
\mathcal{C}:|z-a|=\frac{1}{2}\left(\Delta-B+|a|-\varepsilon \sqrt{(\Delta-B-|a|)^{2}-4 B^{2}}\right), \quad-1<\varepsilon<1
$$

the following condition holds true,

$$
\sup _{z \in \mathcal{C}} \frac{\left|\Phi_{\mathbf{n}}(z)-a\right|}{|z-a|}<1
$$

and $\Phi_{\mathbf{n}}(z)$ and all functions $G_{\mathbf{n}}(z ; \mathbf{m})$ are analytic inside and on $\mathcal{C}$.

Proof: It follows from the estimate in (4.22) that the condition in (4.27) is implied by

$$
\frac{B^{2}}{\Delta-B-|z-a|}+|a|<|z-a| .
$$

The latter holds true if and only if

$$
(|z-a|-(\Delta-B+|a|))^{2}<(\Delta-B-|a|)^{2}-4 B^{2},
$$

which has non-trivial solutions as in (4.26) provided that $2 B<\Delta-B-|a|$. This proves (4.27) under the given assumptions. The statements concerning analyticity are implied by Proposition 4.2.3.

Remark 4.2.5 The best possible bound in (4.22) is obviously obtained for $b=0$, but we need $b>0$ to prove square integrability of the eigenfunctions.

To show that Hypothesis 4.2.1 is relevant we now discuss a few special cases where it holds true.

Lemma 4.2.6 Let $N=2$ and $\lambda$ be non-integer. Then Hypothesis 4.2.1 holds true with

$$
a=0, \quad \Delta=\min _{\nu \in \mathbb{Z}^{\prime}}\left|2 \nu\left(\nu+n_{1}-n_{2}+\lambda\right)\right|>0 \quad \text { if } \lambda \notin \mathbb{Z} \quad(N=2) .
$$

Proof: This is a simple consequence of Equation (3.10).

It is interesting to note that $\Delta$ in (4.28) can be arbitrarily large, e.g. for $n_{1}-n_{2}+\lambda=k+r$, $k \in \mathbb{N}$ and $0<r<1 / 2, \Delta$ can be as large as $2 r k$. It thus is possible to find cases where $\Delta$ is large enough for our series to converge for all values $q<1$.

The reason why we did not find a simple, general proof of convergence are the resonances discussed at the end of Section 3, as can be seen by the following result: 
Lemma 4.2.7 Let $N \geq 2$ and $\lambda$ and $\mathbf{n} \in \mathbb{Z}^{N}$ such that the no-resonance condition in (3.9) is satisfied. Then Hypothesis 4.2.1 holds true for $a=0$ and some $\Delta>0$.

Proof: Since the set of all $\mathbf{m}$ such that $\left|\mathcal{E}_{0}(\mathbf{m})-\mathcal{E}_{0}(\mathbf{n})\right|<1$ (say) is obviously finite,

$$
\Delta \stackrel{\text { def }}{=} \inf _{\mathbf{m}}\left|\mathcal{E}_{0}(\mathbf{m})-\mathcal{E}_{0}(\mathbf{n})\right|
$$

can be computed as minimum over a finite set, and thus (3.9) implies $\Delta>0$.

Note that the no-resonance condition is satisfied in all cases $\mathbf{n}$ where there is no $\lambda$ independent resonance and where $\lambda$ is irrational. Another interesting special case where Lemma 4.2.7 applies is the groundstate $\mathbf{n}=\mathbf{0}$ and irrational $\lambda$.

Remark 4.2.8 The careful reader might wonder why our estimates in Proposition 4.2.3 are not analytic in $q^{2}$. The reason is that these estimates are not optimal. Indeed, since $\Phi_{\mathbf{n}}(z)=0$ for $q=0$ (see Remark 4.1.3), we expect that $\Phi_{\mathbf{n}}(z)$ should vanish like $q^{2}$ as $q \rightarrow 0$. In Remark C.2.1 (Appendix) we motivate the

$$
\text { Conjecture: } K_{s}(\mathbf{0}) \leq \tilde{B}^{s} q^{2\left\lceil\frac{s}{N}\right\rceil} \text { with } \tilde{B} \stackrel{\text { def }}{=} \frac{N(N-1)|\gamma|}{\left(1-q^{2 / N}\right)^{3}}
$$

This would imply the following improved estimate

$$
\text { Conjecture: }\left|\Phi_{\mathbf{n}}(z)\right| \leq \tilde{B} q^{2}\left(\frac{(\Delta-|z-a|)}{\left[(\Delta-|z-a|)^{N}-\tilde{B}^{N} q^{2}\right]} \sum_{n=1}^{N-1} \tilde{B}^{N-n}(\Delta-|z-a|)^{n}-1\right)
$$

analytic in $q^{2}$ and consistent with $\Phi_{\mathbf{n}}(z)=\mathcal{O}\left(q^{2}\right)$. We expect there exists a similar improved estimate for $G_{\mathbf{n}}(z ; \mathbf{m})$.

\subsection{Explicit solution}

From the implicit equation for $\mathcal{E}_{\mathbf{n}}$ in Theorem 4.1.2 it is straightforward to get an explicit expression by expanding in $\gamma$ and thus extend the series solution in Section 3 to all orders. It is important to note that this amounts to an expansion around $\mathcal{E}=\mathcal{E}_{0}(\mathbf{n})$. As will be shown below, it is possible to expand around any point $\mathcal{E}=\mathcal{E}_{0}(\mathbf{n})+a, a$ arbitrary, and thus the 'resonance denominators' $\mathcal{E}_{0}(\mathbf{m})-\mathcal{E}_{0}(\mathbf{n})$ can be moved to $\mathcal{E}_{0}(\mathbf{m})-\mathcal{E}_{0}(\mathbf{n})-a$. In this way the resonance problem can be circumvented, and we obtain the following fully explicit result.

Theorem 4.3.1 (A) Let $\mathbf{n} \in \mathbb{Z}^{N}$ and $a \in \mathbb{R}$ such that the condition in (4.18) holds true for some $\Delta>0$. Then the eigenvalue equation $H \Psi(\mathbf{x})=\mathcal{E} \Psi(\mathbf{x})$ of the eCS differential operator in (1.1)-(1.2) has a solution $\mathcal{E}=\mathcal{E}_{\mathbf{n}}$ as follows,

$$
\begin{aligned}
\mathcal{E}_{\mathbf{n}}= & \mathcal{E}_{0}(\mathbf{n})+a+\sum_{m=1}^{\infty} \sum_{\ell_{0}, \ell_{1}, \ldots, \ell_{m-1}=0}^{\infty} \delta\left(\sum_{r=0}^{m-1} \ell_{r}, m\right) \\
& \times \delta\left(\sum_{r=1}^{m-1} r \ell_{r}, m-1\right)(m-1) ! \prod_{r=0}^{m-1} \frac{\left[\Phi_{\mathbf{n}}^{(r)}(a)\right]^{\ell_{r}}}{\ell_{r} !}
\end{aligned}
$$


with

$$
\begin{aligned}
\Phi_{\mathbf{n}}^{(r)}(a) & =-\sum_{s=2}^{\infty} \gamma^{s} \sum_{\substack{\hat{\boldsymbol{\nu}}_{1}, \ldots, \hat{\boldsymbol{\nu}}_{s} \\
\kappa=1}}^{s} \prod_{\nu_{\kappa}} \sum_{k_{1}, k_{2}, \ldots, k_{s-1}=0}^{\infty} \delta\left(\sum_{\kappa=1}^{s-1} k_{\kappa}, r\right) \\
\times & \frac{\delta\left(\mathbf{0}, \sum_{\kappa=1}^{s} \hat{\boldsymbol{\nu}}_{\kappa}\right)}{\prod_{\kappa=1}^{s-1}\left[\left[\mathcal{E}_{0}\left(\mathbf{n}+\sum_{\ell=1}^{\kappa} \hat{\boldsymbol{\nu}}_{\ell}\right)-\mathcal{E}_{0}(\mathbf{n})-a\right]\right]_{\mathbf{n}}^{1+k_{\kappa}}}-\delta_{r, 0} a
\end{aligned}
$$

and the notation defined in (2.6), (2.7) and (4.9). Moreover, the coefficients $\alpha_{\mathbf{n}}(\mathbf{m})$ giving the corresponding eigenfunction $\Psi(\mathbf{x})=\Psi_{\mathbf{n}}(\mathbf{x})$ according to Theorem 4.1 .2 are

$$
\begin{aligned}
\alpha_{\mathbf{n}}(\mathbf{m})=G_{\mathbf{n}}^{(0)}(a ; \mathbf{m})+ & \sum_{m=1}^{\infty} \sum_{\ell=0}^{m-1} \sum_{\ell_{0}, \ell_{1}, \ldots, \ell_{\ell}=0}^{\infty} \delta\left(\sum_{r=0}^{\ell} \ell_{r}, m\right) \delta\left(\sum_{r=1}^{\ell} r \ell_{r}, \ell\right) \\
& \times(m-1) !(m-\ell) G_{\mathbf{n}}^{(m-\ell)}(a ; \mathbf{m}) \prod_{r=0}^{\ell} \frac{\left[\Phi_{\mathbf{n}}^{(r)}(a)\right]^{\ell_{r}}}{\ell_{r} !}
\end{aligned}
$$

with $\Phi_{\mathbf{n}}^{(r)}(a)$ as above and

$$
\begin{aligned}
G_{\mathbf{n}}^{(r)}(a ; \mathbf{m})=\delta_{r, 0} \delta(\mathbf{m}, \mathbf{n}) & +\sum_{s=1}^{\infty} \gamma^{s} \sum_{\hat{\boldsymbol{\nu}}_{1}, \ldots, \hat{\boldsymbol{\nu}}_{s}} \prod_{r=1}^{s} S_{\nu_{r}} \sum_{k_{1}, k_{2}, \ldots, k_{s}=0}^{\infty} \delta\left(\sum_{\kappa=1}^{s} k_{\kappa}, r\right) \\
& \times \frac{\delta\left(\mathbf{m}, \mathbf{n}+\sum_{\kappa=1}^{s} \hat{\boldsymbol{\nu}}_{\kappa}\right)}{\prod_{\kappa=1}^{s}\left[\left[\mathcal{E}_{0}\left(\mathbf{n}+\sum_{\ell=1}^{\kappa} \hat{\boldsymbol{\nu}}_{\ell}\right)-\mathcal{E}_{0}(\mathbf{x})-a\right]\right]_{\mathbf{n}}^{1+k_{\kappa}}} .
\end{aligned}
$$

The results above hold true for arbitrary parameter values, and $\mathcal{E}_{\mathbf{n}}$ and $\alpha_{\mathbf{n}}(\mathbf{m})$ above are independent of the parameter a in the sense of formal power series.

(B) If $\Delta>|a|$ and if $q$ is small enough that $B$ in (4.20) satisfies the condition $B<$ $(\Delta-|a|) / 3$, then all series above converge absolutely, the following estimates holds true,

$$
\left|\mathcal{E}_{\mathbf{n}}-\mathcal{E}_{0}(\mathbf{n})-a\right| \leq \frac{1}{2}\left(\Delta-B+|a|-\sqrt{(\Delta-B-|a|)^{2}-4 B^{2}}\right)
$$

and

$$
\left|\alpha_{\mathbf{n}}(\mathbf{m})\right| \leq \delta(\mathbf{m}, \mathbf{n})+q^{\sum_{j} j K\left(m_{j}-n_{j}\right)} \frac{2 B}{\Delta-B-|a|+\sqrt{(\Delta-B-|a|)^{2}-4 B^{2}}},
$$

with $K$ in (2.20), and the eigenfunction $\Psi_{\mathbf{n}}(\mathbf{x})$ determined by the formulas above is square integrable.

Proof: We observe that (4.14) is of the form $z=\eta \varphi(z)$ if we identify

$$
z=\tilde{\mathcal{E}}_{\mathbf{n}}, \quad \eta \varphi(z)=\Phi_{\mathbf{n}}(z) .
$$

We thus can use Lagrange's theorem 2.4.1 to solve (4.14). Note that $\eta$ can be regarded as a book keeping parameter which can be set to any value. We set $\eta=1$ in the following. 
We first prove the result under the assumptions stated in (B). According to Corollary 4.2.4 the prerequisites for Lagrange's theorem are then fulfilled for any of the contours defined in (4.26). Thus the equation $z=\Phi_{\mathbf{n}}(z)$ has a single, simple solution $z=\xi$ within any such contour. Taking the infimum over the allowed $\varepsilon$-values we obtain the estimate in (4.34). Moreover, using (2.22) for $g(z)=z$ we obtain the following explicit formula for the eigenvalues by an absolutely convergent series,

$$
\mathcal{E}_{\mathbf{n}}=\mathcal{E}_{0}(\mathbf{n})+\xi \text { with } \xi=a+\sum_{m=1}^{\infty} \frac{1}{m !} \frac{\mathrm{d}^{m-1}}{\mathrm{~d} a^{m-1}} \varphi(a)^{m} .
$$

We now can write the equation in (4.16) as

$$
\alpha_{\mathbf{n}}(\mathbf{m})=g(\xi), \quad g(z)=G_{\mathbf{n}}(z ; \mathbf{m})
$$

where $g(z)$ fulfills the prerequisites of Lagrange's theorem 2.4.1 according to Corollary 4.2.4. Thus (2.22) gives an explicit formula for the $\alpha_{\mathbf{n}}(\mathbf{m})$ by an absolutely convergent series. Moreover, inserting (4.34) in the estimate in (4.23) we obtain (4.35).

To make this series more explicit we insert (2.23) and use the following multinomial series,

$$
\begin{array}{r}
\left(\sum_{r=0}^{\infty} \varphi_{r}(z-a)^{r}\right)^{m}=\sum_{\ell_{0}, \ell_{1}, \ldots=0}^{\infty} \delta\left(\sum_{r=0}^{\infty} \ell_{r}, m\right) \frac{m !}{\prod_{r=0}^{\infty} \ell_{r} !} \prod_{r=0}^{\infty}\left[\varphi_{r}\right]^{\ell_{r}}(z-a)^{\sum_{r=0}^{\infty} r \ell_{r}}= \\
\sum_{\ell=0}^{\infty}(z-a)^{\ell} \sum_{\ell_{0}, \ldots, \ell_{\ell}=0}^{\infty} \delta\left(\sum_{r=0}^{\ell} \ell_{r}, m\right) \delta\left(\sum_{r=1}^{\ell} r \ell_{r}, \ell\right) \frac{m !}{\prod_{r=0}^{\ell} \ell_{r} !} \prod_{r=0}^{\ell}\left[\varphi_{r}\right]^{\ell_{r}}
\end{array}
$$

and similarly for $g$. This yields

$$
\xi=a+\sum_{m=1}^{\infty} \sum_{\ell_{0}, \ell_{1}, \ldots, \ell_{m-1}=0}^{\infty} \delta\left(\sum_{r=0}^{m-1} \ell_{r}, m\right) \delta\left(\sum_{r=1}^{m-1} r \ell_{r}, m-1\right)(m-1) ! \prod_{r=0}^{m-1} \frac{\left[\varphi_{r}\right]^{\ell_{r}}}{\ell_{r} !}
$$

and

$$
\begin{gathered}
g(\xi)=g(a)+\sum_{m=1}^{\infty} \sum_{\ell=0}^{m-1} \sum_{\ell_{0}, \ell_{1}, \ldots, \ell_{\ell}=0}^{\infty} \delta\left(\sum_{r=0}^{\ell} \ell_{r}, m\right) \\
\times \delta\left(\sum_{r=1}^{\ell} r \ell_{r}, \ell\right)(m-1) !(m-\ell) g_{m-\ell} \prod_{r=0}^{\ell} \frac{\left[\varphi_{r}\right]^{\ell_{r}}}{\ell_{r} !} .
\end{gathered}
$$

We observe that

$$
\varphi_{r}=\frac{1}{r !} \frac{\mathrm{d}^{r}}{\mathrm{~d} a^{r}}\left(\Phi_{\mathbf{n}}(a)-a\right) \stackrel{\text { def }}{=} \Phi_{\mathbf{n}}^{(r)}(a) .
$$

Recalling (4.36) and inserting (4.15) we obtain (4.30) and (4.31); we used

$$
\frac{1}{r !} \frac{\mathrm{d}^{r}}{\mathrm{~d} a^{r}} \frac{1}{\prod_{\kappa=1}^{s}\left(x_{\kappa}-a\right)}=\sum_{k_{1}, \ldots, k_{s}=0}^{\infty} \delta\left(\sum_{\kappa=1}^{s} k_{\kappa}, r\right) \frac{1}{\prod_{\kappa=1}^{s}\left(x_{\kappa}-a\right)^{1+k_{\kappa}}}
$$


for complex parameters $x_{\kappa}$, which follows from the Leibniz rule. Similarly, recalling

$$
g_{r}=\frac{1}{r !} \frac{\mathrm{d}^{r}}{\mathrm{~d} a^{r}} G_{\mathbf{n}}(a ; \mathbf{m}) \stackrel{\text { def }}{=} G_{\mathbf{n}}^{(r)}(a ; \mathbf{m})
$$

and (4.37) and using (4.17) we obtain (4.32) and (4.33).

The estimates in (4.35) and Lemma 2.3.1 imply

$$
\begin{array}{r}
\left|\Psi_{\mathbf{n}}(\mathbf{x})\right| \leq \sum_{\mathbf{m}}\left|\alpha_{\mathbf{n}}(\mathbf{m})\right|\left|f_{\mathbf{m}}(\mathbf{z})\right|<\sum_{\mathbf{m}}\left(\delta(\mathbf{m}, \mathbf{n})+q^{\sum_{j} j K\left(m_{j}-n_{j}\right)} \tilde{C}\right) \times \\
C q^{\sum_{j}\left(\tilde{K}\left|m_{j}\right|-K j m_{j}\right)}=C q^{-\sum_{j} K j n_{j}}\left(q^{\sum_{j} \tilde{K}\left|n_{j}\right|}+\tilde{C}\left(\frac{1+q^{\tilde{K}}}{1-q^{\tilde{K}}}\right)^{N}\right)
\end{array}
$$

for all $b>0$, with $\tilde{C}=2 B /\left[\Delta-B-|a|+\sqrt{(\Delta-B-|a|)^{2}-4 B^{2}}\right]$ and the constants in $(2.20)$ and (4.20). This proves that $\Psi_{\mathbf{n}}(\mathbf{x})$ is uniformly bounded, and thus square integrable, on its domain $[-\pi, \pi]^{N}$. This completes the proof of (B).

If we only know that $a$ is such that (4.18) holds true for some $\Delta>0$ we cannot conclude anything about convergence. However, the results stated in (A) still hold true in the sense of formal power series; see Theorem 2.4.1 and Remark 2.4.2,

Remark 4.3.2 Since $\Phi_{\mathbf{n}}(z)$ is proportional to $q^{2}$ (see Remark 4.2.8) one can choose the parameter $\eta$ in (4.36) to be $q^{2}$, and this shows that the $m$-th term in (4.30) and (4.32) is $\mathcal{O}\left(q^{2 m}\right)$. From this we expect that these series have a finite radius of convergence in $q^{2}$ in general. However, since the function $\varphi(z)$ also depends on $q^{2}$, this is not easy to prove.

Remark 4.3.3 The result in Theorem 4.3.1 explains why the power series of $\mathcal{E}_{\mathbf{n}}$ in $q^{2}$ is very complicated: due to (3.11) the term $\propto \gamma^{s}$ on the r.h.s in (4.15) is $\mathcal{O}\left(q^{2\left[\frac{s}{N}\right\rceil}\right)$; see Remark C.2.1 in the Appendix. Thus all such terms $\propto \gamma^{s}$ with $s=2, \ldots, \ell N$ contribute to the power series coefficient $\propto q^{2 \ell}$ of $\mathcal{E}_{\mathbf{n}}$, and the complexity of this $q^{2 \ell}$-term therefore increases dramatically not only with $\ell$ but also with $N$.

Remark 4.3.4 Our proof of convergence relies on estimates in Section 4.2 which are crude. In particular, we estimate the energy denominators $\left[\mathcal{E}_{0}(\mathbf{m})-\mathcal{E}_{0}(\mathbf{n})-z\right]$ in (4.15) and (4.17) by their smallest possible value which, if resonances exist, are assumed for resonances. However, resonances are rare, and it is easy to see that there always exists a constant $\Delta_{0}>0$ such that $\left|\mathcal{E}_{0}(\mathbf{m})-\mathcal{E}_{0}(\mathbf{n})\right|>\Delta_{0}$ for all $\mathbf{m}$ different from a resonance. It thus seemed to us that it should be easy to improve our estimates in Proposition 4.2 .3 enough to prove convergence of our series for arbitrary particle numbers $N$. However, despite of much effort (delaying the publication of this paper for two more years) we have not been able to do this up to now. Anyway, we have several independent reasons to believe in the existence of a finite radius of convergence for arbitrary parameters: (i) the results in [KT], (ii) the existence of variants of our solution method described in Section 4.4 and Remark 2 in Section $[5$ which both avoid resonances, (iii) recent numerical results [BL]]. 


\subsection{A method to avoid resonances}

We now describe a generalization of our solution described in Sections 4.14 4.3 which allows to avoid resonances. The idea is to use a variant of degenerate perturbation theory.

We fix $\mathbf{n} \in \mathbb{Z}^{N}$ and consider the set of all corresponding resonances, i.e. all $\mathbf{m} \in \mathbb{Z}^{N}$ different from $\mathbf{n}$ such that $\sum_{j}\left(m_{j}-n_{j}\right)=0$ and $\mathcal{E}_{0}(\mathbf{m})=\mathcal{E}_{0}(\mathbf{n})$. It is easy to see that there can be only a finite number of distinct resonances which we denote as $\mathbf{n}_{2}, \mathbf{n}_{3}, \ldots, \mathbf{n}_{R+1}$

with $R$ the number of resonances. We also set $\mathbf{n}_{1} \stackrel{\text { def }}{=} \mathbf{n}$. For example, for $N=2$ and integer $\lambda$ we get $R=1, \mathbf{n}_{1}=\left(n_{1}, n_{2}\right)$ and $\mathbf{n}_{2}=\left(n_{2}-\lambda, n_{1}+\lambda\right)$; see (3.10).

We now apply Lemma 4.1.1 to (4.1) using the following projection, $\left(\mathbb{P}_{\mathbf{n}} \alpha\right)(\mathbf{m}) \stackrel{\text { def }}{=} \alpha(\mathbf{m})$ for $\mathbf{m}=\mathbf{n}_{J}, J=1,2, \ldots, R+1$, and 0 otherwise; $\mathbb{A}$ and $\mathbb{B}$ are as before. The general solution of (4.6) is now $\alpha_{0}=\sum_{J=1}^{R+1} c_{J} \delta_{\mathbf{n}_{J}}$ with constants $c_{J}$ to be determined. This implies the following generalization of (4.10),

$$
\Psi_{\mathbf{n}}(\mathbf{x})=\sum_{J=1}^{n} \sum_{\mathbf{m} \in \mathbb{Z}^{N}} c_{J} \alpha_{\mathbf{n}_{J}}(\mathbf{m}) \hat{F}_{\mathbf{m}}(\mathbf{x})
$$

with $\alpha_{\mathbf{n}_{J}}(\mathbf{m})$ obtained by setting $\mathbf{n}=\mathbf{n}_{J}$ in (4.11) and

$$
\frac{1}{\left[\left[\mathcal{E}_{0}(\mathbf{m})-\mathcal{E}\right]\right]_{\mathbf{n}}} \stackrel{\text { def }}{=} \begin{cases}0 & \text { if } \mathbf{m}=\mathbf{n}_{J} \text { for } J=1,2, \ldots, R+1 \\ {\left[\mathcal{E}_{0}(\mathbf{m})-\mathcal{E}\right]^{-1}} & \text { otherwise } .\end{cases}
$$

The equation determining the eigenvalues $\mathcal{E}_{\mathbf{n}}=\mathcal{E}_{0}(\mathbf{n})+\tilde{\mathcal{E}}_{\mathbf{n}}$ and the coefficients $c_{J}$ is obtained from (4.7) which can be written as follows,

$$
\tilde{\mathcal{E}}_{\mathbf{n}} c_{J}=\sum_{K=1}^{R+1} \Phi_{\mathbf{n}_{J}, \mathbf{n}_{K}}\left(\tilde{\mathcal{E}}_{\mathbf{n}}\right) c_{K}
$$

with

$$
\Phi_{\mathbf{n}_{J}, \mathbf{n}_{K}}(z)=-\sum_{s=1}^{\infty} \gamma^{s} \sum_{\hat{\boldsymbol{\nu}}_{1}, \ldots, \hat{\boldsymbol{\nu}}_{s}} \prod_{\kappa=1}^{s} S_{\nu_{\kappa}} \frac{\delta\left(\mathbf{n}_{J}, \mathbf{n}_{K}+\sum_{\kappa=1}^{s} \hat{\boldsymbol{\nu}}_{\kappa}\right)}{\prod_{\kappa=1}^{s-1}\left[\left[\mathcal{E}_{0}\left(\mathbf{n}_{K}+\sum_{\ell=1}^{\kappa} \hat{\boldsymbol{\nu}}_{\ell}\right)-\mathcal{E}_{0}(\mathbf{n})-z\right]\right]_{\mathbf{n}}} .
$$

It should be possible to solve (4.46) explicitly using a matrix version of Lagrange's reversion theorem. One now can expand about $z=0$, and we expect that our estimates in Section 4.2 can be made sharp enough to prove absolute convergence in a finite $q$-interval without restrictions on parameters. It would be interesting to explore this generalized solution algorithm in more detail, but this is beyond the scope of the present paper.

\section{Final remarks}

1. The functions $\mathcal{J}_{\mathbf{n}}(\mathbf{z})$ defined in (1.10) can be expanded as follows,

$$
\mathcal{J}_{\mathbf{n}}(\mathbf{z})=\sum_{\ell=0}^{\infty} \mathcal{J}_{\ell, \mathbf{n}}(\mathbf{z}) q^{2 \ell}
$$


where $\mathcal{J}_{0, \mathbf{n}}(\mathbf{z})$ are (essentially) the Jack polynomials [McD, St], as discussed in the introduction. It is interesting to note that for partitions $\mathbf{n}$, all functions

$$
\left(z_{1} z_{2} \cdots z_{N}\right)^{\ell} \mathcal{J}_{\mathbf{n}, \ell}(\mathbf{z}), \quad \ell=0,1,2, \ldots
$$

are symmetric polynomials [L1]. It would be interesting to investigate if these polynomials have a combinatorial significance also for $\ell \geq 1$.

2. Proposition 2.2.1 above suggest that the function in (2.1) has an expansion in eigenfunctions $\Psi_{\mathbf{n}}(\mathbf{x})$ of the eCS Hamiltonian as follows,

$$
F(\mathbf{x} ; \mathbf{y})=\sum_{\mathbf{n}} \kappa_{\mathbf{n}} \Psi_{\mathbf{n}}(\mathbf{x}) \overline{\Psi_{\mathbf{n}}(\mathbf{y})}
$$

for some constants $\kappa_{\mathbf{n}}$ and the bar indicating complex conjugation, and our algorithm provides a means to extract from this the eigenfunctions.

It is well-known that the eCS model has a family of $N$ independent (formally) selfadjoint differential operators of the form $H_{k}=(-\mathrm{i})^{N} \sum_{j=1}^{N} \frac{\partial^{k}}{\partial x_{j}^{k}}+$ (lower order terms) for $k=$ $1,2, \ldots N$ which mutually commute, $\left[H_{k}, H_{\ell}\right]=0$ for all $k, \ell=1,2, \ldots, N$, and including the total momentum operator $P=H_{1}$ and the eCS Hamiltonian $H=H_{2}$ in (1.1) [OP]. This suggests that the remarkable identity should be generalizable to all these differential operators,

$$
\text { Conjecture: } \quad\left[H_{k}(\mathbf{x})-H_{k}(\mathbf{y})\right] F(\mathbf{x} ; \mathbf{y})=0 \text { for all } k
$$

(for $k=1$ the proof is trivial). Using this one could extend our results to the operator $\sum_{k=1}^{N} b_{k} H_{k}$ for arbitrary real coefficients $b_{k}$. The resulting formulas would be similar to ours but with $\mathcal{E}_{0}(\mathbf{n})$ in $(2.13)$ replaced by the $\sum_{j, k=1}^{N} b_{k}\left(n_{j}+\frac{\lambda}{2}(N+1-2 j)\right)^{k}$. The freedom to choose the parameters $b_{j}$ arbitrarily should allow to avoid the resonance problem completely, similarly as in [KT]. It would be interesting to find a direct proof of the identities in (5.4) and to derive and explore the explicit solution obtained with the operator $\sum_{k=1}^{N} b_{k} H_{k}$.

3. To set our results in perspective we note that a formal series representation of eigenfunctions and eigenvalues of the kind derived in this paper can be given for any quantum mechanical model. Indeed, assume that we want to diagonalize some self-adjoint Hilbert space operator $H$ using some countable generating set $f_{n}$ of the pertinent Hilbert space such that

$$
H f_{n}=E_{n} f_{n}+\sum_{m} V_{n m} f_{m} \quad\left(V_{n n}=0\right)
$$

for some constants $E_{n}$ and $V_{n m}$. For example, if $H$ is a Hamiltonian and $f_{n}$ a complete orthonormal basis then $\left\langle f_{n}, H f_{m}\right\rangle=E_{n}$ for $m=n$ and $V_{n m}$ otherwise, but what we describe here is equally true for any generating sets which are not orthonormal and/or are overcomplete. It is easy to see that our arguments in Section 4 can be generalized to the present general case and imply that $H$ has formal eigenfunctions

$$
\psi_{n}=\sum_{m} \alpha_{n}(m) f_{m}
$$


with corresponding formal eigenvalues $\mathcal{E}_{n}=E_{n}+\tilde{\mathcal{E}}_{n}$ where $\tilde{\mathcal{E}}_{n}$ is a solution of the equation $\tilde{\mathcal{E}}_{n}=\Phi_{n}\left(\tilde{\mathcal{E}}_{n}\right)$ with

$$
\Phi_{n}(z)=\sum_{s=1}^{\infty}(-1)^{s} \sum_{k_{1}, k_{2}, \ldots, k_{s}} \frac{V_{n k_{1}} V_{k_{1} k_{2}} \cdots V_{k_{s} n}}{\prod_{r=1}^{s}\left[\left[E_{k_{r}}-E_{n}-z\right]\right]_{n}}
$$

where $1 /\left[\left[E_{k}-z\right]\right]_{n}=1 /\left[E_{k}-z\right]$ for $k \neq n$ and 0 for $k=n$. Moreover, the coefficients for these eigenfunctions are given by

$$
\alpha_{n}(m)=\delta_{n m}+\sum_{s=1}^{\infty}(-1)^{s} \sum_{k_{0}, k_{1}, \ldots, k_{s}} \frac{\delta_{n, k_{0}}\left(\prod_{r=1}^{s} V_{k_{r-1, k_{r}}}\right) \delta_{k_{s}, m}}{\prod_{r=1}^{s}\left[\left[E_{k_{r}}-E_{n}-\tilde{\mathcal{E}}_{n}\right]\right]_{n}}
$$

(this result is known as Brillouin-Wigner perturbation theory; see e.g. [Lö] and references therein). Using the formula in (2.22) one can obtain from this fully explicit formulas for the eigenvalues and eigenfunctions as formal series to all orders; see the formulas given in the proof of Theorem 4.1.2. This shows that it is not the existence of an explicit series solution which makes the eCS model special, but it is rather the details of this solution. To be more specific, the eCS model is special since there exists a basis $f_{n}$ such that $E_{n}$ and $V_{n m}$ are given by simple explicit formulas, that $V_{n m}$ only depends on $n-m$, and that the matrix $V_{n m}$ is close to triangular which implies that the series solution converges (the latter property we only proved here in special cases, but we believe that it is true in general).

It is worth mentioning that the formulas above shed some interesting light on quantum mechanical perturbation theory in general. In particular, they highlight that, for a given Hamiltonian $H$, it is the choice of the basis $f_{n}$ which determines the usefulness of perturbation theory. Moreover, the formulas above hold true for any linear operator $H$ and can be easily generalized to degenerate cases as described in Section 4.4.

4. Our algorithm was based on the remarkable identity in (2.5) which we first obtained using quantum field theory techniques [L1, L2]. While we latter found an elementary proof of this result (presented in Appendix A.3), we feel that the quantum field theory proof is more illuminating since it not only shows that this result it true but also why. We also note that there exist interesting generalizations of this identity which we found using these quantum field theory results [L6].

5. To judge the usefulness of our results it is important study our series solution numerically. Numerical results for $N=2$ were recently obtained by J.C. Barba and show that the numerical convergence is much better than the estimates in Section 4.2 suggest [BL]. To be more specific, it seems that resonances are no problem in practice, and low order truncations of our series approximate the exact solution well not only for small $q$-values but up to values close to $q=1$ and for a wide range of coupling parameters.

6. For $\lambda=1, q=0$, and partitions $\mathbf{n}$ the functions $f_{\mathbf{n}}(\mathbf{z})$ in $(2.9)$ are identical with the Schur polynomials [HL]. Our results therefore include simple explicit formulas for an elliptic deformation of the Schur polynomials which seem worth to be studied in more detail.

7. We believe that the solution method presented in this paper can be extended to other examples, including the anharmonic oscillator and the Heun equation 5 We hope to come

\footnotetext{
${ }^{5}$ This was suggested to us by Vadim Kuznetsov.
} 
back to this in future work.

Acknowledgments. This work owes much to Vadim Kuznetsov and is therefore dedicated to his memory. I am also grateful to J.C. Barba, M. Hallnäs, G. Lindblad, J. Mickelsson, A. Polychronakos, S.G. Rajeev, S. Ruijsenaars, and E. Sklyanin for helpful comments and to J.C. Barba and M. Hallnäs for reading the manuscript. I thank F. Calogero, B. Kupershmidt, N. Nekrasov, Y. Suris, S. Rauch, E. Sklyanin, and P. Winternitz for their interest and encouragement. We also acknowledge financial support by the Swedish Science Research Council (VR), the Göran Gustafsson Foundation, and the European Union through the FP6 Marie Curie RTN ENIGMA (Contract number MRTN-CT-2004-5652).

\section{A Identities of elliptic functions}

In this Appendix we give an elementary proof of the Fact stated in (2.1) $-(2.2)$ and on which our algorithm is based. For the convenience of the reader we also include the proofs of some properties of elliptic functions which we need.

\section{A.1 Relation of $V$ and $\wp$}

Here we prove (1.2) and (1.6).

From the definition in (1.2) it is obvious that $V(z), z \in \mathbb{C}$, is doubly periodic with periods $2 \omega_{1}=2 \pi$ and $2 \omega_{2}=\mathrm{i} \beta$, it has a single pole of order 2 in each period-parallelogram, $V(z)-z^{-2}$ is analytic in some neighborhood of $z=0$ and equal to $c_{0}$ in (1.3); we used $q=\exp \left(\pi \mathrm{i} \omega_{2} / \omega_{1}\right)=\exp (-\beta / 2)$. These facts imply the second identity in (1.2) (see e.g. [EMOT], Section 13.12).

To prove (1.6) we note that $\theta(2 z)$ equals, up to a constant, the Jacobi Theta function $\vartheta_{1}(z):$

$$
\theta(z)=\frac{1}{2 q^{1 / 4} \prod_{n=1}^{\infty}\left(1-q^{2 n}\right)} \vartheta_{1}(z / 2)
$$

(see e.g. page 470 in [WW]). From the relation between $\vartheta_{1}$ and the Weierstrass elliptic functions $\sigma, \zeta$ and $\wp$ we therefore conclude (see e.g. page 473 in [WW])

$$
\zeta(z)=\frac{d}{d z} \log \theta(z)+\frac{\eta_{1} z}{\omega_{1}}
$$

and

$$
\wp(z)=-\frac{d}{d z} \zeta(z)=-\frac{d^{2}}{d z^{2}} \log \theta(z)-\frac{\eta_{1}}{\omega_{1}}
$$

where $\eta_{1} / \omega_{1}$ is a constant. To determine the latter constant we use the definition in (1.5) and compute

$$
\log \theta(z)=\log \left[(z / 2)-(z / 2)^{3} / 6\right]+\sum_{n=1}^{\infty} \log \left[\left(1-q^{2 n}\right)^{2}+q^{2 n} z^{2}\right]+\mathcal{O}\left(z^{4}\right)=
$$




$$
\text { const. }+\log (z)-\left(\frac{1}{24}-\sum_{n=1}^{\infty} \frac{q^{2 n}}{\left(1-q^{2 n}\right)^{2}}\right) z^{2}+\mathcal{O}\left(z^{4}\right) \text {. }
$$

Recalling that $\wp(z)-z^{-2}$ vanishes for $z \rightarrow 0$ one concludes from this and (A.3) that $\eta_{1} / \omega_{1}=c_{0}$, recalling (1.3). This together with (1.2) and (1.3) proves (1.6).

\section{A.2 Proof of the identities in $(2.3)-(2.4)$}

We start with the following well-known identity for the Weierstrass elliptic functions $\zeta$ and $\wp$,

$$
[\zeta(x)+\zeta(y)+\zeta(z)]^{2}=\wp(x)+\wp(y)+\wp(z) \quad \text { if } x+y+z=0
$$

(this identity is given as an exercise on page 446 in [WW]). From (A.2) we conclude that $\phi(x)=\theta^{\prime}(x) / \theta(x)$ equals $\zeta(x)$ up to a term linear in $x$. Thus the identity in (A.4) remains true if we replace $\zeta$ by $\phi$. This together with the trivial identity

$$
\begin{array}{r}
\phi(x) \phi(y)+\phi(x) \phi(z)+\phi(y) \phi(z)= \\
=\frac{1}{2}[\phi(x)+\phi(y)+\phi(z)]^{2}-\frac{1}{2}\left[\phi(x)^{2}+\phi(y)^{2}+\phi(z)^{2}\right]
\end{array}
$$

implies (2.3).

\section{A.3 Proof of Lemma 2.1.1}

Let $F=F(\mathbf{x} ; \mathbf{y})$ as defined in (2.1). We compute

$$
\frac{\partial}{\partial x_{j}} F=\left[\sum_{k \neq j} \lambda \phi\left(x_{j}-x_{k}\right)-\sum_{k} \lambda \phi\left(x_{j}-y_{k}\right)\right] F
$$

with $\phi(x)=\theta^{\prime}(x) / \theta(x)$, and thus

$$
\begin{array}{r}
\frac{\partial^{2}}{\partial x_{j}^{2}} F=\left[\sum_{k \neq j} \lambda \phi^{\prime}\left(x_{j}-x_{k}\right)-\sum_{k} \lambda \phi^{\prime}\left(x_{j}-y_{k}\right)+\right. \\
\sum_{k, \ell \neq j} \lambda^{2} \phi\left(x_{j}-x_{k}\right) \phi\left(x_{j}-x_{\ell}\right)+\sum_{k, \ell} \lambda^{2} \phi\left(x_{j}-y_{k}\right) \phi\left(x_{j}-y_{\ell}\right) \\
\left.-2 \sum_{k \neq j, \ell} \lambda^{2} \phi\left(x_{j}-x_{k}\right) \phi\left(x_{j}-y_{\ell}\right)\right] F .
\end{array}
$$

With that we compute straightforwardly

$$
W \stackrel{\text { def }}{=} \frac{1}{F} \sum_{j=1}^{N}\left(\frac{\partial^{2}}{\partial x_{j}^{2}}-\frac{\partial^{2}}{\partial y_{j}^{2}}\right) F
$$

which we write as a sum of four terms, $W=W_{1}+W_{2}+W_{3}+W_{4}$, with

$$
W_{1} \stackrel{\text { def }}{=} \sum_{j} \sum_{k \neq j}\left[\lambda \phi^{\prime}\left(x_{j}-x_{k}\right)+\lambda^{2} \phi\left(x_{j}-x_{k}\right)^{2}\right]-[x \leftrightarrow y]
$$


(' $[x \leftrightarrow y]$ ' means the same terms but with the arguments $x_{j}$ and $y_{j}$ interchanged),

$$
\begin{gathered}
W_{2} \stackrel{\text { def }}{=} \sum_{k, j}\left[-\lambda \phi^{\prime}\left(x_{j}-y_{k}\right)+\lambda^{2} \phi\left(x_{j}-y_{k}\right)^{2}\right]-[x \leftrightarrow y], \\
W_{3} \stackrel{\text { def }}{=} \sum_{j} \sum_{k \neq j} \sum_{\ell \neq j, k}\left[\lambda^{2} \phi\left(x_{j}-x_{k}\right) \phi\left(x_{j}-x_{\ell}\right)\right]-[x \leftrightarrow y],
\end{gathered}
$$

and

$$
\begin{array}{r}
W_{4} \stackrel{\text { def }}{=} \sum_{j, k} \sum_{\ell \neq k}\left[\lambda^{2} \phi\left(x_{j}-y_{k}\right) \phi\left(x_{j}-y_{\ell}\right)-2 \lambda^{2} \phi\left(x_{k}-x_{\ell}\right) \phi\left(x_{k}-y_{j}\right)\right]-[x \leftrightarrow y]= \\
\sum_{j, k} \sum_{\ell \neq k}\left[\lambda^{2} \phi\left(x_{j}-y_{k}\right) \phi\left(x_{j}-y_{\ell}\right)+2 \lambda^{2} \phi\left(y_{k}-y_{\ell}\right) \phi\left(y_{k}-x_{j}\right)\right]-[x \leftrightarrow y] .
\end{array}
$$

We first observe that the first two terms in $W_{2}$ are invariant under $x \leftrightarrow y$ [note that $\left.\phi^{\prime}(-x)=\phi^{\prime}(x)\right]$, and therefore

$$
W_{2}=0 .
$$

We then write $W_{3}$ as follows [using $\phi(-x)=-\phi(x)$ ]

$$
\begin{array}{r}
W_{3}=\sum_{j<k<\ell}\left(-\lambda^{2}\right)\left[\phi\left(x_{k}-x_{j}\right) \phi\left(x_{j}-x_{\ell}\right)+\phi\left(x_{\ell}-x_{k}\right) \phi\left(x_{k}-x_{j}\right)+\right. \\
\left.\phi\left(x_{j}-x_{\ell}\right) \phi\left(x_{\ell}-x_{k}\right)\right]-[x \leftrightarrow y],
\end{array}
$$

and using now the relation in (2.3) and $f(-x)=f(x)$ we get

$$
\begin{array}{r}
W_{3}=\sum_{j<k<\ell}\left(-\lambda^{2}\right)\left[f\left(x_{k}-x_{j}\right)+f\left(x_{j}-x_{\ell}\right)+f\left(x_{\ell}-x_{k}\right)\right]-[x \leftrightarrow y] \\
=\sum_{j} \sum_{k \neq j} \sum_{\ell \neq j, k}\left[-\lambda^{2} f\left(x_{j}-x_{k}\right)\right]-[x \leftrightarrow y] \\
=-(N-2) \lambda^{2} \sum_{j} \sum_{k \neq j}\left[f\left(x_{j}-x_{k}\right)-f\left(y_{j}-y_{k}\right)\right] .
\end{array}
$$

Finally,

$$
\begin{array}{r}
W_{4}=\sum_{j, k} \sum_{\ell \neq k}\left(-\lambda^{2}\right)\left[\phi\left(y_{k}-x_{j}\right) \phi\left(x_{j}-y_{\ell}\right)+\phi\left(y_{\ell}-y_{k}\right) \phi\left(y_{k}-x_{j}\right)+\right. \\
\left.\phi\left(y_{\ell}-y_{k}\right) \phi\left(x_{j}-y_{\ell}\right)\right]-[x \leftrightarrow y]
\end{array}
$$

where we wrote the same term in two different ways by renaming summation indices. We can now use the relation in (2.3) again, and we obtain

$$
\begin{array}{r}
W_{4}=\sum_{j, k} \sum_{\ell \neq k}\left(-\lambda^{2}\right)\left[f\left(y_{k}-x_{j}\right)+f\left(x_{j}-y_{\ell}\right)+f\left(y_{\ell}-y_{k}\right)\right]-[x \leftrightarrow y] \\
N \lambda^{2} \sum_{j} \sum_{k \neq j}\left[f\left(x_{j}-x_{k}\right)-f\left(y_{j}-y_{k}\right)\right]
\end{array}
$$


where the terms even under $[x \leftrightarrow y]$ canceled. Collecting all terms and using $\phi^{\prime}(x)=-V(x)$ and $2 f(x)=V(x)-\phi(x)^{2}+c_{0}$ we get

$$
\begin{array}{r}
W=\sum_{j} \sum_{k \neq j}\left[\lambda \phi^{\prime}\left(x_{j}-x_{k}\right)+\lambda^{2} \phi\left(x_{j}-x_{k}\right)^{2}-\lambda^{2}(N-2) f\left(x_{j}-x_{k}\right)\right. \\
\left.+\lambda^{2} N f\left(x_{j}-x_{k}\right)\right]-[x \leftrightarrow y]= \\
\sum_{j} \sum_{k \neq j}\left[\lambda \phi^{\prime}\left(x_{j}-x_{k}\right)+\lambda^{2} \phi\left(x_{j}-x_{k}\right)^{2}+2 \lambda^{2} f\left(x_{j}-x_{k}\right)\right]-[x \leftrightarrow y]= \\
\sum_{j} \sum_{k \neq j}\left[\lambda(\lambda-1) V\left(x_{j}-x_{k}\right)-\lambda(\lambda-1) V\left(y_{j}-y_{k}\right)\right] .
\end{array}
$$

We thus see that $W F$ is equal to the r.h.s. of (2.2).

\section{B Proof of Proposition 2.2.1}

We first observe that Lemma 2.1.1 remains true if we replace $F(\mathbf{x} ; \mathbf{y})$ by

$$
F^{\prime}(\mathbf{x} ; \mathbf{y})=c \mathrm{e}^{\mathrm{i} P \sum_{j=1}^{N}\left(x_{j}-y_{j}\right)} F(\mathbf{x} ; \mathbf{y})
$$

for arbitrary constants $P \in \mathbb{R}$ and $c \in \mathbb{C}$. [To see this, introduce center-of-mass coordinates $X=\sum_{j=1}^{N} x_{j} / N$ and $x_{j}^{\prime}=\left(x_{j}-x_{1}\right)$ for $j=2, \ldots, N$, and similarly for the $y$ 's. Then $H(\mathbf{x})=-\partial^{2} / \partial X^{2}+H_{c}\left(\mathbf{x}^{\prime}\right)$, and similarly for $H(\mathbf{y})$. Invariance of (2.2) under $F \rightarrow \exp [-\mathrm{i} P(X-Y) N] F$ thus follows from $(\partial / \partial X+\partial / \partial Y) F(\mathbf{x} ; \mathbf{y})=0$, and the latter is implied by the obvious invariance of $F(\mathbf{x} ; \mathbf{y})$ under $x_{j} \rightarrow x_{j}+a, y_{j} \rightarrow y_{j}+a, a \in \mathbb{R}$. Invariance of (2.2) under $F \rightarrow c F$ is trivial, of course].

As shown below, one can find constants $c$ and $P$ such that the function $F^{\prime}(\mathbf{x} ; \mathbf{y})$ above is the generating function for the $\hat{F}_{\mathbf{n}}(\mathbf{x})$ in (2.8) as follows,

$$
F^{\prime}(\mathbf{x} ; \mathbf{y})=\sum_{\mathbf{n} \in \mathbb{Z}} \hat{F}_{\mathbf{n}}(\mathbf{x}) \xi_{1}^{-n_{1}^{+}} \xi_{2}^{-n_{2}^{+}} \cdots \xi_{N}^{-n_{N}^{+}}, \quad \xi_{j}=\mathrm{e}^{\mathrm{i} y_{j}}
$$

with

$$
n_{j}^{+}=n_{j}+\frac{\lambda}{2}(N+1-2 j),
$$

and the series converges absolutely in the following region,

$$
1<\left|\xi_{1}\right|<\left|\xi_{2}\right|<\cdots<\left|\xi_{N}\right|<q^{-2}
$$

Moreover, in the same region one can change variables in the eCS Hamiltonian $H(\mathbf{y})$ from $y_{j}$ to $\xi_{j}$ and expand

$$
H(\mathbf{y})=\sum_{j}\left(\xi_{j} \frac{\partial}{\partial \xi_{j}}\right)^{2}-\gamma \sum_{j<k} \sum_{\nu \in \mathbb{Z}} S_{\nu}\left(\frac{\xi_{j}}{\xi_{k}}\right)^{\nu}
$$


with the coefficients $S_{\nu}$ in (2.14). Inserting this in $H(\mathbf{x}) F^{\prime}(\mathbf{x} ; \mathbf{y})=H(\mathbf{y}) F^{\prime}(\mathbf{x} ; \mathbf{y})$ (implied by Lemma (2.1.1) ) and equating the coefficients of $\xi_{1}^{-n_{1}^{+}} \xi_{2}^{-n_{2}^{+}} \cdots \xi_{N}^{-n_{N}^{+}}$on both sides we obtains the identity in (2.12) with $\mathcal{E}_{0}(\mathbf{n})=\sum_{j}\left(n_{j}^{+}\right)^{2}$ as in (2.13).

To prove (B.2) we note that the definition of the functions $f_{\mathbf{n}}(\mathbf{z})$ in $(2.9)$ is equivalent to the following generating function,

$$
\frac{\prod_{1 \leq j<k \leq N} \Theta\left(\xi_{j} / \xi_{k}\right)}{\prod_{j, k=1}^{N} \Theta\left(z_{j} / \xi_{k}\right)}=\sum_{\mathbf{n} \in \mathbb{Z}} f_{\mathbf{n}}(\mathbf{z}) \xi_{1}^{-n_{1}} \xi_{2}^{-n_{2}} \cdots \xi_{N}^{-n_{N}}
$$

Moreover, using

$$
\theta(y)=\frac{1}{2} \mathrm{e}^{\mathrm{i} \pi / 2} \xi^{-1 / 2} \Theta(\xi) \quad \text { for } \xi=\mathrm{e}^{\mathrm{i} y} \text { if }|\xi|<1
$$

following from (1.5) and the obvious identity $\sin (y / 2)=\frac{1}{2} \mathrm{e}^{\mathrm{i} \pi / 2} \mathrm{e}^{-\mathrm{i} y / 2}\left(1-\mathrm{e}^{\mathrm{i} y}\right)$, we find by a straightforward computation that $F(\mathbf{x} ; \mathbf{y})$ in (2.1) is equal to the expression on the l.h.s. of (B.6) multiplied by $\Psi_{0}(\mathbf{x})$ in (1.9) and the factor

$$
\mathrm{e}^{\mathrm{i} \pi \lambda\left[N(N-1) / 2-N^{2}\right] / 2} \frac{\prod_{j<k} \mathrm{e}^{-\mathrm{i} \lambda\left(y_{j}-y_{k}\right) / 2}}{\prod_{j, k} \mathrm{e}^{-\mathrm{i} \lambda\left(x_{j}-y_{k}\right) / 2}}=\text { const. } \mathrm{e}^{\mathrm{i} \lambda N \sum_{j=1}^{N}\left(x_{j}-y_{j}\right) / 2} \mathrm{e}^{-\mathrm{i} \lambda \sum_{j=1}^{N}(N+1-2 j) y_{j} / 2} .
$$

This shows that (B.2) holds true for certain constants $P$ and $c$. We are left to prove

$$
V(y)=-\sum_{\nu \in \mathbb{Z}} S_{\nu} \xi^{-\nu} \text { for } \xi=\mathrm{e}^{\mathrm{i} y} \text { such that }|\xi|<1
$$

which obviously implies (B.5). For that we insert the identity

$$
\frac{1}{4 \sin ^{2}[(\varphi \pm \mathrm{i} \varepsilon) / 2]}=-\sum_{\nu=1}^{\infty} \nu \mathrm{e}^{ \pm \mathrm{i} \nu \varphi-\nu \varepsilon} \text { for all real } \varphi \text { and } \varepsilon>0
$$

in (1.2) and obtain $V(y)=-\sum_{\nu=1}^{\infty} \nu\left[\xi^{-\nu}+\sum_{m=1}^{\infty} \mathrm{e}^{-m \beta}\left(\xi^{\nu}+\xi^{-\nu}\right)\right]$. Summing up the geometric series in $m$ we obtain (B.7).

\section{Further proofs}

\section{C.1 Proof of Lemma 2.3.1}

We first prove the estimates in (2.18) using the definition in (2.11):

$$
|\Theta(z)| \leq(1+|z|) \prod_{m=1}^{\infty}\left[\left(1+q^{2 m}|z|\right)\left(1+q^{2 m} /|z|\right)\right]=\Theta(-|z|),
$$

and

$$
|\Theta(z)| \geq(1-|z|) \prod_{m=1}^{\infty}\left[\left(1-q^{2 m}|z|\right)\left(1-q^{2 m} /|z|\right)\right]=\Theta(|z|)
$$


for $q^{2} \leq|z| \leq 1$. With that we can estimate the integrand in (2.9) as follows,

$$
\left|\left(\prod_{j} \xi_{j}^{n_{j}}\right) \frac{\prod_{j<k} \Theta\left(\xi_{j} / \xi_{k}\right)^{\lambda}}{\prod_{j, k} \Theta\left(\mathrm{e}^{\mathrm{i} x_{j}} / \xi_{k}\right)^{\lambda}}\right| \leq\left(\prod_{j}\left|\xi_{j}^{n_{j}}\right|\right) \frac{\prod_{j<k} \Theta\left(-\left|\xi_{j} / \xi_{j}\right|\right)^{\lambda}}{\prod_{j, k} \Theta\left(1 /\left|\xi_{k}\right|\right)^{\lambda}}
$$

where the r.h.s. is constant on the integration paths in (2.10). Thus (2.9) implies the following upper bound,

$$
\left|f_{\mathbf{n}}(\mathbf{z})\right| \leq \mathrm{e}^{\sum_{j} \varepsilon_{j} n_{j}} \frac{\prod_{j<k} \Theta\left(-\mathrm{e}^{-\left(\varepsilon_{k}-\varepsilon_{j}\right)}\right)^{\lambda}}{\prod_{j, k} \Theta\left(\mathrm{e}^{-\varepsilon_{k}}\right)^{\lambda}}
$$

for any choice of allowed parameters $\varepsilon_{j}$. To make the bound in (C.1) more specific we choose

$$
\varepsilon_{j}=\varepsilon\left(j-\rho \operatorname{sign}\left(n_{j}\right)\right), \quad \varepsilon=\frac{\beta}{N+b}, \quad \rho=\frac{b}{1+2 b}
$$

with $b>0$ arbitrary; note that these parameters satisfy the conditions in (2.10). We also observe that

$$
\frac{1}{2} \Theta(-x)<\frac{\Theta(-x)}{1+x}<\frac{\Theta\left(-q^{2}\right)}{1+q^{2}}<\Theta\left(-q^{2}\right) \text { for } q^{2}<x<1
$$

and

$$
\frac{\Theta(x)}{1-x_{1}} \geq \frac{\Theta(x)}{1-x} \geq \frac{\Theta\left(x_{0}\right)}{1-x_{0}}>0 \text { for } q^{2}<x_{0} \leq x \leq x_{1}<1
$$

these estimates are easily proved using $\Theta( \pm x) /(1 \mp x)=\prod_{n=1}^{\infty}\left[1+q^{4 n} \mp q^{2 n}(x+1 / x)\right]$ and the fact that the function $x+1 / x$ is monotonically decreasing in the interval $0<x<1$. Thus

$$
\left|f_{\mathbf{n}}(\mathbf{z})\right|<\mathrm{e}^{\sum_{j} \varepsilon\left(j n_{j}-\rho\left|n_{j}\right|\right)} \frac{\left[2 \Theta\left(-q^{2}\right)\right]^{N(N-1) \lambda / 2}}{\left[\left(1-x_{1}\right) \Theta\left(x_{0}\right) /\left(1-x_{0}\right)\right]^{N^{2} \lambda}}
$$

with $x_{0}=\min _{k, \mathbf{n}} \mathrm{e}^{-\varepsilon_{k}}=\mathrm{e}^{-\varepsilon(N+\rho)}$ and $x_{1}=\max _{k, \mathbf{n}} \mathrm{e}^{-\varepsilon_{k}}=\mathrm{e}^{-\varepsilon(1-\rho)}$. Inserting $\mathrm{e}^{-\varepsilon}=q^{K}$ and $\rho K=\tilde{K}$ we obtain $x_{0}=q^{2-2 b \tilde{K}}$ and $x_{1}=q^{K-\tilde{K}}$.

\section{C.2 Proof of the estimates in (4.25)}

In this appendix we derive the estimate (4.25) for the sum $K_{s}(\mathbf{m})$ defined in (4.24).

Representing the Kronecker delta by integrals,

$$
\delta\left(\mathbf{m}, \sum_{\kappa=1}^{s} \hat{\boldsymbol{\nu}}_{\kappa}\right)=\left(\prod_{j=1}^{N} \int_{-\pi}^{\pi} \frac{d y_{j}}{2 \pi}\right) \mathrm{e}^{-\mathrm{i} \mathbf{y} \cdot\left(\mathbf{m}-\sum_{\kappa} \hat{\boldsymbol{\nu}}_{\kappa}\right)}
$$

and recalling (2.7), $\mathbf{y} \cdot \hat{\boldsymbol{\nu}}_{\kappa}=\nu_{\kappa}\left(y_{j_{\kappa}}-y_{k_{\kappa}}\right)$, we obtain

$$
K_{s}(\mathbf{m})=|\gamma|^{s}\left(\prod_{j=1}^{N} \int_{-\pi}^{\pi} \frac{d y_{j}}{2 \pi} \mathrm{e}^{-\left(\mathrm{i} y_{j}+\varepsilon_{j}\right) m_{j}}\right)\left(\sum_{1 \leq j<k \leq N} \sum_{\nu \in \mathbb{Z}} S_{\nu} \mathrm{e}^{\mathrm{i} \nu\left(y_{j}-y_{k}\right)-\nu\left(\varepsilon_{k}-\varepsilon_{j}\right)}\right)^{s}
$$


for $0<\varepsilon_{1}<\varepsilon_{2}<\cdots<\varepsilon_{N}<\beta$ in the limit $\varepsilon_{N} \downarrow 0$. The shifts in the integration paths, $y_{j} \rightarrow y_{j}-\mathrm{i} \varepsilon_{j}$, are to guarantee the absolute convergence of the sums in the integrand. It it important to note that, actually, this limit need not be taken: changing variables to $\xi_{j}=\mathrm{e}^{\mathrm{i} y_{j}+\varepsilon_{j}}$ the latter integral can be written as follows,

$$
K_{s}(\mathbf{m})=|\gamma|^{s}\left(\prod_{j=1}^{N} \oint_{\mathcal{C}_{j}} \frac{\mathrm{d} \xi_{j}}{2 \pi \mathrm{i} \xi_{j}} \xi_{j}^{-m_{j}}\right)\left(\sum_{j<k} \sum_{\nu \in \mathbb{Z}} S_{\nu}\left(\frac{\xi_{j}}{\xi_{k}}\right)^{\nu}\right)^{s}
$$

with the integration paths $\mathcal{C}_{j}$ defined in (2.10). The integrand is analytic for all $\varepsilon_{j}$ obeying the condition in (2.10), as can be checked by recalling the definition of $S_{\nu}$ in (2.14) and $q^{2}=\mathrm{e}^{-\beta}$. Thus Cauchy's theorem implies that integral on the r.h.s. in (C.2) is independent of the $\varepsilon_{j}$.

Inserting $\varepsilon_{j}=j \varepsilon$ where $0<\varepsilon<\beta / N$ and using the triangle inequality and $S_{\nu} \geq 0$ we deduce from (C.2) that

$$
\begin{array}{r}
\left|K_{s}(\mathbf{m})\right| \leq|\gamma|^{s}\left(\prod_{j=1}^{N} \int_{-\pi}^{\pi} \frac{d y_{j}}{2 \pi}\left|\mathrm{e}^{-\left(\mathrm{i} y_{j}+j \varepsilon\right) m_{j}}\right|\right)\left(\sum_{j<k} \sum_{\nu \in \mathbb{Z}} S_{\nu}\left|\mathrm{e}^{\left.\mathrm{i} \nu\left(y_{j}-y_{k}\right)-\nu(k-j) \varepsilon\right)}\right|\right)^{s} \\
=\mathrm{e}^{-\sum_{j} j \varepsilon m_{j}}\left(|\gamma| \sum_{j<k} \sum_{\nu \in \mathbb{Z}} S_{\nu} \mathrm{e}^{-\nu(k-j) \varepsilon}\right)^{s} .
\end{array}
$$

To simplify this bound we use $S_{\nu} \leq|\nu| q^{|\nu|-\nu} /\left(1-q^{2}\right)$ following from the definition in (2.14) and thus

$$
\begin{array}{r}
\sum_{j<k} \sum_{\nu \in \mathbb{Z}} S_{\nu} \mathrm{e}^{-\nu(k-j) \varepsilon} \leq \sum_{j<k} \sum_{\nu \in \mathbb{Z}}|\nu| \frac{q^{|\nu|-\nu}}{\left(1-q^{2}\right)} \mathrm{e}^{-\nu(k-j) \varepsilon}= \\
\frac{1}{\left(1-q^{2}\right)} \sum_{j<k} \sum_{\nu=1}^{\infty} \nu\left(\mathrm{e}^{-\nu(k-j) \varepsilon}+\mathrm{e}^{-\nu[\beta-(k-j) \varepsilon]}\right)= \\
\frac{1}{\left(1-q^{2}\right)} \sum_{\ell=1}^{N-1} \sum_{\nu=1}^{\infty} \nu\left((N-\ell) \mathrm{e}^{-\nu \ell \varepsilon}+\ell \mathrm{e}^{-\nu[\beta-(N-\ell) \varepsilon]}\right)= \\
\frac{1}{\left(1-q^{2}\right)} \sum_{\ell=1}^{N-1}\left((N-\ell) \frac{\mathrm{e}^{-\ell \varepsilon}}{\left(1-\mathrm{e}^{-\ell \varepsilon}\right)^{2}}+\ell \frac{\mathrm{e}^{-[\beta-N \varepsilon]-\ell \varepsilon}}{\left(1-\mathrm{e}^{-[\beta-N \varepsilon]-\ell \varepsilon}\right)^{2}}\right)
\end{array}
$$

where we used $q^{2}=\mathrm{e}^{-\beta}$ and changed summation variables. Inserting the estimate

$$
\frac{p}{(1-p x)^{2}}<\frac{1}{(1-x)^{2}} \quad \text { if } 0<p<1 \text { and } 0<p x^{2}<1
$$

for $p=\exp (-[\beta-N \varepsilon])$ and $x=\exp (-\varepsilon)$ we get

$$
\begin{array}{r}
\sum_{j<k} \sum_{\nu \in \mathbb{Z}} S_{\nu} \mathrm{e}^{-\nu(k-j)}<\frac{N}{\left(1-q^{2}\right)} \sum_{\ell=1}^{N-1} \frac{\mathrm{e}^{-\ell \varepsilon}}{\left(1-\mathrm{e}^{-\ell \varepsilon}\right)^{2}}< \\
\frac{N}{\left(1-q^{2}\right)} \sum_{\ell=1}^{N-1} \frac{\mathrm{e}^{-\ell \varepsilon}}{\left(1-\mathrm{e}^{-\varepsilon}\right)^{2}}<\frac{N(N-1) \mathrm{e}^{-\varepsilon}}{\left(1-\mathrm{e}^{-\varepsilon}\right)^{3}}
\end{array}
$$


since $\left(1-\mathrm{e}^{-(N-1) \varepsilon}\right)<\left(1-q^{2}\right)$. To summarize,

$$
K_{s}(\mathbf{m})<\mathrm{e}^{-\sum_{j} j \varepsilon m_{j}}\left(\frac{|\gamma| N(N-1) \mathrm{e}^{-\varepsilon}}{\left(1-\mathrm{e}^{-\varepsilon}\right)^{3}}\right)^{s} \text { if } 0<\varepsilon<\frac{\beta}{N} .
$$

Setting $\varepsilon=\beta /(N+b)$ for $b>0$ we get the bounds in (4.25).

Remark C.2.1 It is instructive to see in more details how the upper bounds for $K_{s}(\mathbf{0})$ come about: It is easy to see that the contributions of lowest order in $q^{2}$ come from the identity in (3.11), for example for $s \leq N$ the lowest order contributions are for $\hat{\boldsymbol{\nu}}_{r}=-\mathbf{E}_{j_{r} j_{r+1}}$ for $r=1,2, \ldots, s-1$ and $\hat{\boldsymbol{\nu}}_{s}=\mathbf{E}_{j_{1} j_{s}}$. Thus for $s \leq N, K_{s}(\mathbf{0})=c_{N, s}|\gamma|^{s}\left(S_{-1}\right)^{s-1} S_{1}+$ $\ldots=c_{N, s}|\gamma|^{s} q^{2}+\ldots$ with the dots indicating higher order terms, and, more generally, $K_{s}(\mathbf{0})=c_{N, s}|\gamma|{ }^{s} q^{2\left\lceil\frac{s}{N}\right\rceil}+\mathcal{O}\left(q^{2\left\lceil\frac{s}{N}\right\rceil+2}\right)$; the constants $c_{N, s}$ are combinatorial factors. This and (C.4) for $\varepsilon=\beta / N$ and $\mathbf{m}=\mathbf{0}$ suggest the improved estimate in (4.29).

\section{References}

[BL] Barba J.C. and Langmann E. (work in progress)

[B] Billey E.: Algebraic nested Bethe ansatz for the elliptic Ruijsenaars model. arXiv:math/9806068

[C1] Calogero F.: Solution of the one-dimensional N-body problems with quadratic and/or inversely quadratic pair potentials. J. Math. Phys. 12, 419 (1971)

[C2] Calogero F.: Exactly solvable one-dimensional many-body problems. Lett. Nuovo Cimento 13, 411 (1975)

[CL] Carey A.L. and Langmann E.: Loop groups, anyons and the Calogero-Sutherland model. Comm. Math. Phys. 201, 1 (1999)

[DI] Dittrich J. and Inozemtsev V.I.: On the structure of eigenvectors of the multidimensional Lamé operator. J. Phys. A: Math. Gen. 26, L753 (1993)

[EMOT] Erdélyi A., Magnus W., Oberhettinger F., and Tricomi F.G.: Higher Transcendental Functions. Vol. 2, New York-Toronto-London: McGraw-Hill Book Company, Inc. (1953)

[EK] Etingof P.I. and Kirillov A.A.: Representation of affine Lie algebras, parabolic differential equations and Lamé functions. Duke Math. J. 74, 585 (1994)

[FGP] Fernández Núñez J., García Fuertes W., and Perelomov A.M.: A perturbative approach to the quantum elliptic Calogero-Sutherland model. Phys. Lett. A 307, 233 (2003)

[FV1] Felder G. and Varchenko A.: Integral representation of solutions of the elliptic Knizhnik-Zamolodchikov-Bernard equations. Int. Math. Res. Notices 5, 221 (1995) 
[FV2] Felder G. and Varchenko A.: Three formulas for eigenfunctions of integrable Schroedinger operators. arXiv: hep-th/9511120

[FV3] Felder G. and Varchenko A.: Elliptic quantum groups and Ruijsenaars models. arXiv:q-alg/9704005

[GGR] Gómez-Ullate D., González-López A., and Rodríguez M.Á.: Exact solutions of an elliptic Calogero-Sutherland model. Phys. Lett. B 511, 112 (2001)

[HL] Hallnäs M. and Langmann E.: Quantum Calogero-Sutherland type models and generalised classical polynomials, arXiv:math-ph/0703090

[HSY] Hou B.Y, Sasaki R., and Yang W.-L.: Eigenvalues of Ruijsenaars-Schneider models associated with $\mathrm{A}_{n-1}$ root system in Bethe ansatz formalism. J. Math. Phys. 45, 559 (2004)

[I1] Inozemtsev V.I.: Solution to three-magnon problem for $S=1 / 2$ periodic quantum spin chains with elliptic exchange. J. Math. Phys. 37, 147 (1996)

[I2] Inozemtsev V.I.: The eigenvectors of the Heisenberg Hamiltonian with elliptic form of the exchange spin interaction. J. Nonlin. Math. Phys. 12, 395 (2005)

[KR] Komori Y., and Ruijsenaars S.N.M.: Elliptic integrable systems of Calogero-Moser type: A survey. In: Proceedings of the 2004 Kyoto Workshop on Elliptic integrable systems, Noumi M., and Takasaki K., eds. Rokko Lectures in Math., Dept. of Math., Kobe Univ., pp. 201-221

[KT] Komori Y., and Takemura K.: The perturbation of the quantum Calogero-MoserSutherland system and related results. Comm. Math. Phys. 227, 93 (2002)

[KMS] Kuznetsov V.B., Mangazeev V.V., and Sklyanin E.K.: Q-operator and factorised separation chain for Jack polynomials. Indag. Math. 14, 451 (2003)

[KS] Kuznetsov V.B. and Sklyanin E.K.: On Bäcklund transformations for many-body systems. J. Phys. A: Math. Gen. 31, 2241 (1998)

[L1] Langmann E.: Anyons and the elliptic Calogero-Sutherland model. Lett. Math. Phys. 54, $279(2000)$

[L2] Langmann E.: Second quantization of the elliptic Calogero-Sutherland model. Comm. Math. Phys. 247, 321 (2004)

[L3] Langmann E.: Algorithms to solve the (quantum) Sutherland model. J. Math. Phys. 42, $4148(2001)$

[L4] Langmann E.: An algorithm to solve the elliptic Calogero-Sutherland model. arXiv:math-ph/0401029 v1 and v2]

[L5] Langmann E.: An explicit solution of the (quantum) elliptic Calogero-Sutherland model, in: "Symmetry and Perturbation Theory (SPT04)", Eds.: G. Gaeta et.al., World Scientific (2005), 159 arXiv:math-ph/0407050 
[L6] Langmann E.: Remarkable identities related to the (quantum) elliptic CalogeroSutherland model. J. Math. Phys. 47, 022101 (2006)

[L7] Langmann E.: A method to derive explicit formulas for an elliptic generalization of the Jack polynomials. In: "Jack, Hall-Littlewood and Macdonald polynomials", V.B. Kuznetsov and S. Sahi (eds.), Contemporary Mathematics 417, American Mathematical Society, p. 257 (2006) arXiv:math-ph/0511015

[Lö] Löwdin P.-O.: A note on quantum-mechanical perturbation theory, J. Chem. Phys. 19, $1396(1951)$

[McD] Macdonald I.G.: Symmetric functions and Hall polynomials. Oxford Mathematical Monographs. Oxford: Clarendon Press 1979

[OP] Olshanetsky M.A. and Perelomov A.M.: Quantum completely integrable systems connected with semisimple Lie algebras. Lett. Math. Phys. 2, 7 (1977)

[RS] Reed M. and Simon B.: Methods of Modern Mathematical Physics Vol. II. New York, London: Academic Press 1975

[R1] Ruijsenaars S.N.M.: Complete integrability of relativistic Calogero-Moser systems and elliptic function identities. Comm. Math. Phys. 110, 191 (1987)

[R2] Ruijsenaars S.N.M.: Generalized Lamé functions. I. The elliptic case. J. Math. Phys. 40, 1595 (1999)

[S] Sklyanin E.K.: Separation of variables. New trends. Prog. Theor. Phys. Suppl. 118, 35 (1995)

[St] Stanley R.P.: Some properties of Jack symmetric functions. Adv. in Math. 77, 76 (1989)

[Su1] Sutherland B.: Exact results for a quantum many body problem in one-dimension. II. Phys. Rev. A5, 1372 (1972)

[Su2] Sutherland B.: Exact ground state wave function for a one-dimensional plasma. Phys. Rev. Lett. 34, 1083 (1975)

[T] Takemura K.: On the eigenstates of the elliptic Calogero-Moser model. Lett. Math. Phys. 53, 181 (2000)

[WW] Whittaker E.T. and Watson G.N.: Course of modern analysis. 4th edition. Cambridge Univ. Press (1958)

[W] Wojciechowski S.: The analogue of the Bäcklund transformation for integrable manybody systems. J. Phys. A: Math. Gen. 15, L653 (1982) 